\title{
Compactness Theorem for Some Generalized Second-Order Language
}

\author{
Zakharov V. K. ${ }^{1}$ \& Yashin A. D. ${ }^{2}$ \\ ${ }^{1}$ Chair of Theoretical Information, Faculty of Mechanics and Mathematics, Lomonosov Moscow State University \\ (Lomonosov MSU), Moscow, Russia \\ ${ }^{2}$ Chair of Applied Mathematics, Faculty of Information Technologies, Moscow State University of Psychology \\ and Education (MSUPE), Moscow, Russia \\ Correspondence: Zakharov V. K., Faculty of Mathematics and Mechanics, Lomonosov Moscow State University, \\ Leninskie Gory, Moscow 119992, Russia. Tel: 7-495-939-1303. E-mail: zakharov_valeriy@list.ru
}

Received: March 21, 2014 Accepted: April 22, 2014 Online Published: June 26, 2014

doi:10.5539/jmr.v6n3p21 URL: http://dx.doi.org/10.5539/jmr.v6n3p21

\begin{abstract}
For the first-order language the compactness theorem was proved by K. Gödel and A. I. Mal'cev in 1936. In 1955, it was proved by J. Łoś (1955) by means of the method of ultraproducts. Unfortunately, for the usual second-order language the compactness theorem does not hold. Moreover, the method of ultraproducts is also inapplicable to second-order models. A possible way out of this situation is to refuse the most vulnerable place in the construction of ultraproducts connected with the factorization relatively an ultrafilter, i.e., to stay working with the ordinary non factorized product. It compels us instead of the single usual set-theoretical equality = to use several generalized equalities $\approx_{\text {first }}$ and $\approx_{\text {second }}$ for first and second orders, and instead of the single usual set-theoretical belonging

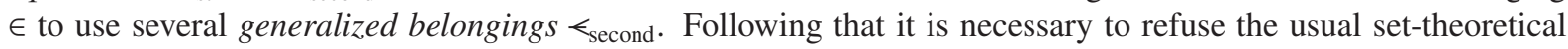
interpretation $\left(\gamma\left(x_{0}\right), \ldots, \gamma\left(x_{k}\right)\right) \in \gamma(u)$ of the second basic (after equality) atomic formula $\left(x_{0}, \ldots, x_{k}\right) u$ and to replace it by the generalized interpretation $\left(\gamma\left(x_{0}\right), \ldots, \gamma\left(x_{k}\right)\right)<_{\tau} \gamma(u)$, where $x_{i}^{\tau_{i}}$ are variables of the first-order types $\tau_{i}, u^{\tau}$ is a variable of the second-order type $\tau=\left[\tau_{0}, \ldots, \tau_{k}\right]$ (i.e. predicate), and $\gamma$ is some evaluation of variables on some mathematical system $U$.

This paper is devoted to rigorous development of the expressed general idea. For the generalized in such a manner second-order language the compactness theorem is proved by means of the method of infraproducts consisting in rejection of the Łoś factorization. In the end of the paper the method of infraproducts is applied for the construction of some uncountable models of the second-order generalized Peano-Landau arithmetic.
\end{abstract}

Keywords: second-order language, generalized equality, generalized belonging, infraproduct, infrafiltration, compactness, Peano-Landau arithmetics

\section{Introduction}

For the first-order language the compactness theorem was proved by K. Gödel and A. I. Mal'cev in 1936 (see, e.g., Ershov \& Palyutin, 1984; §17; Mal'cev, 1970, 8.3; Tourlakis, 2003, 1.5.42). In 1955 it was proved by J. Loś (1955) by means of the method of ultraproducts (see also Ershov \& Palyutin, 1984, §17; Mendelson, 1997, 2.14).

Unfortunately, for the usual second-order language (see, e.g., Mal'cev, 1970, §6; Mendelson, 1997, Appendix; Takeuti, 1975, §16) the compactness theorem does not hold (see, e.g., Mendelson, 1997, Appendix; Boolos \& Jeffrey, 1989, §18). Moreover, the method of ultraproducts is also inapplicable to second-order models.

A possible way out of this situation is to refuse the most vulnerable place in the construction of ultraproducts connected with the factorization relatively an ultrafilter, i.e., to stay working with the ordinary non factorized product. This refusal compels us instead of the single usual set-theoretical equality $=$ to use several generalized equalities $\approx_{\text {first }}$ and $\approx_{\text {second }}$ for first and second orders, and instead of the single usual set-theoretical belonging

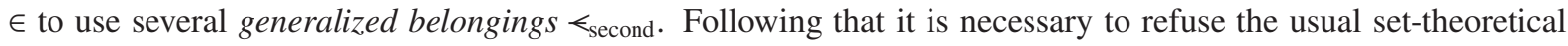
interpretation $\left(\gamma\left(x_{0}\right), \ldots, \gamma\left(x_{k}\right)\right) \in \gamma(u)$ of the second basic (after equality) atomic formula $\left(x_{0}, \ldots, x_{k}\right) u$ and to replace it by the generalized interpretation $\left(\gamma\left(x_{0}\right), \ldots, \gamma\left(x_{k}\right)\right)<_{\tau} \gamma(u)$, where $x_{i}^{\tau_{i}}$ are variables of the first-order types 
$\tau_{i}, u^{\tau}$ is a variable of the second-order type $\tau=\left[\tau_{0}, \ldots, \tau_{k}\right]$ (i.e. predicate), and $\gamma$ is some evaluation of variables on some mathematical system $U$.

This paper is devoted to rigorous development of the expressed general idea. A short presentation of this idea was announced in Zakharov (2008).

In capacity of initial formulas the formulas of the following two forms were taken: the formula $y^{\sigma} \delta_{\sigma} z^{\sigma}$ for the generalized equality $\delta_{\sigma}$ and the formula $\left(x_{0}^{\tau_{0}}, \ldots, x_{k}^{\tau_{k}}\right) \varepsilon_{\tau} u^{\tau}$ for the generalized belonging $\varepsilon_{\tau}$, where $y^{\sigma}$ and $z^{\sigma}$ are the variables of the first- or the second-order type $\sigma$ and $x_{i}^{\tau_{i}}$ and $u^{\tau}$ are the variables of the first-order types $\tau_{i}$ and the second-order type $\tau \equiv\left[\tau_{0}, \ldots, \tau_{k}\right]$, respectively.

These atomic formulas are interpreted on an evaluated mathematical system $(U, \gamma)$ (with an evaluation $\gamma$ of variables on $U$ ) in the following generalized way: $\gamma(y) \approx_{\sigma} \gamma(z)$ and $\left(\gamma\left(x_{0}\right), \ldots, \gamma\left(x_{k}\right)\right)<_{\tau} \gamma(u)$, where $\approx_{\sigma}$ is a generalized relation of equality (more exactly, an equivalence relation) and $\epsilon_{\tau}$ is a generalized correspondence of belonging. Generalized equalities and generalized belongings are connected with each other by the initial principle of change of equals (see axiom E4 from section 4).

More exactly, we introduce a generalized second-order signature $\Sigma_{2}^{g}$ containing, in addition to individual and predicate constants and variables, the symbols $\delta_{\tau}$ and $\varepsilon_{\tau}$. With respect to this signature formulas $\varphi$ in the language $L\left(\Sigma_{2}^{g}\right)$ are defined by usual induction, when we start from the above-mentioned atomic formulas.

To give a semantics of the language $L\left(\Sigma_{2}^{g}\right)$ we define mathematical systems $U$ of the signature $\Sigma_{2}^{g}$. The satisfaction of a formula $\varphi$ on a system $U$ with respect to an evaluation of variables $\gamma$ is defined according to the abovementioned generalized interpretation of the atomic formulas (in notation $U \models \varphi[\gamma]$ ).

The semantics for the language $L\left(\Sigma_{2}^{g}\right)$, presented in the given paper, differs both from the standard semantics (see Mendelson, 1997, Appendix; Takeuti, 1975, §16) and from the Henkin semantics (see Mendelson, 1997, Appendix; Takeuti, 1975, §21; Rossberg, 2004; Shapiro, 1991; Väänänen, 2001), which restricts the range of values of the evaluation $\gamma\left(x^{\tau}\right)$ for a variable $x^{\tau}$ of a second-order type $\tau$ by some subset of the power-set $\mathcal{P}(\tau(X))$ of the terminal $\tau(X)$ of the mathematical system $U \equiv(X, S)$.

In the given paper the following generalized compactness theorem is proved:

Let $\Phi$ be a set of formulas of the language $L\left(\Sigma_{2}^{g}\right)$. Let for every finite subset $f$ of the set $\Phi$ there exist a mathematical system $U_{f}$ of the signature $\Sigma_{2}^{g}$ and an evaluation of variables $\gamma_{f}$ on the system $U_{f}$ such that $U_{f}=\varphi\left[\gamma_{f}\right]$ for every formula $\varphi \in f$. Then there exist a mathematical system $U$ of the signature $\Sigma_{2}^{g}$ and an evaluation of variables $\gamma$ on the system $U$ such that $U \vDash \varphi[\gamma]$ for every formula $\varphi \in \Phi$ (see Theorem 2 in section 8).

This system $U$ is constructed with the help of some ultrafilter starting from the systems $U_{f}$ by means of the method of infraproducts consisting in rejection of the Łós factorization.

The most delicate point in the proof of the compactness theorem is the demonstration of the property of infrafiltration for a quantified formula $\exists x^{\tau} \psi$ for a variable $x^{\tau}$ of a second-order type $\tau=\left[\tau_{0} \ldots, \tau_{k}\right]$, which requires some preliminary assertions (see Propositions 2 and 4).

In order to enlarge the area of possible applications of the above-mentioned theorem, it is proved in a polygrade language with basic and auxiliary grades. Therefore interpretations are defined on polygrade domains of the form $\left[A_{0}, \ldots, A_{m} ; K_{0}, \ldots, K_{n-1}\right]$, where $K_{0}, \ldots, K_{n-1}$ are the fixed auxiliary sets (which are absent when $n=0$ ). It allows to consider in capacity of models modules $A_{K}$ over the fixed ring $K$.

The introducing the suite $H \equiv\left[K_{0}, \ldots, K_{n-1}\right]$ of the fixed auxiliary sets requires the introducing the additional condition of H-concordance of mathematical systems $U \equiv(X, S)$ and $V \equiv(Y, T)$, where $S$ and $T$ are the polygrade superstructures over the supports $X \equiv\left[A_{0}, \ldots, A_{m}, K_{0}, \ldots, K_{n-1}\right)$ and $Y \equiv\left[B_{0}, \ldots, B_{m}, K_{o}, \ldots, K_{n-1}\right]$. This condition means the similarity of the systems $U$ and $V$ with respect to all elements of the signature $\Sigma_{2}^{g}$ connnected with the fixed auxiliary suite $H$. Also we use the similar condition of $H$-concordance of an evaluation $\gamma$ on the system $U$ and an evaluation $\delta$ on the system $V$. In turn, this entails the necessity of introducing the additional condition of $H$-concordance in defining the satisfactions $U \models\left(\exists x^{\tau} \varphi\right)[\gamma]$ and $U \models\left(\forall x^{\tau} \varphi\right)[\gamma]$, which is not required for $n=0$, i.e., when the auxiliary suite is absent.

With the exception of the condition of $H$-concordance and some technical difficulties, the polygrade variant $U=$ $\left(\left[A_{0}, \ldots, A_{m} ; K_{0}, \ldots, K_{n-1}\right] ; S\right)$ considered in this paper does not differ in principal from the purely onegrade variant $(A ; S)$. 
In the end of the paper the method of infraproducts is applied for the construction of models of the second-order generalized Peano-Landau arithmetic. The supports of these models are the Baire sets $\mathbb{N}_{0}^{F}$, which are uncountable in general.

Further in the paper the redesignation of a symbol-string $\rho$ by a symbol-string $\sigma$ will be denoted by $\rho \equiv \sigma$ or $\sigma \equiv \rho$.

\section{Types}

For fixed integers $m, n \in \omega_{0}$ define by induction the semitypes and the types:

1) for any $i \in m+1$ the symbol-string $\langle i, 1\rangle$ is the semitype and the type;

2) for any $j \in n$ the symbol-string $\langle j, 0\rangle$ is the semitype and the type;

3 ) if $\tau$ is a type, then $\tau$ is the semitype;

4) if $\tau$ is a semitype, then $[\tau]$ is the type;

5) if $\tau_{0}, \ldots, \tau_{k}$ are semitypes and $k \geq 1$, then $\left(\tau_{o}, \ldots, \tau_{k}\right)$ is the semitype.

This definition is a generalization of the corresponding definition from Takeuti $(1975, \S 20)$.

Further instead of $\left[\left(\tau_{o}, \ldots, \tau_{k}\right)\right]$ we shall write simply $\left[\tau_{o}, \ldots, \tau_{k}\right]$. So the notation $\left[\tau_{o}, \ldots, \tau_{k}\right]$ may be used for $k \geq 0$.

Semantics of semitypes and types will be explained in the next section.

Types $\langle i, 1\rangle$ and $\langle j, 0\rangle$ will be called the first-order types. If $\tau_{0}, \ldots, \tau_{k}$ are first-order types and $k \geq 0$ then $\left[\tau_{0}, \ldots, \tau_{k}\right]$ will be called the second-order type.

For a type $\tau \equiv\left[\tau_{0}, \ldots, \tau_{k}\right]$ with $k \geq 0$ the types $\tau_{0}, \ldots, \tau_{k}$ will be called the parents of the type $\tau$ and will be denoted by $p_{0} \tau, \ldots, p_{k} \tau$, respectively. Consider the set $P(\tau) \equiv\left\{p_{0} \tau, \ldots, p_{k} \tau\right\}$ of all parents of the type $\tau$.

For any first-order type $\tau$ put formally $p \tau \equiv \tau$ and $P(\tau) \equiv\{p \tau\}=\{\tau\}$.

With any type $\tau$ we associate the semitype $\check{\tau}$ of the type $\tau$ as follows:

1) if $\tau$ is a first-order type, then $\check{\tau} \equiv \tau$;

2) if $\tau=\left[\tau_{1}\right]$ and $\tau_{1}$ is a semitype, then $\check{\tau} \equiv \tau_{1}$.

In other words, the semitype of a type is obtained by omitting the square brackets.

An auxiliary type is defined by induction in the following way:

1) any type of the form $\langle j, 0\rangle$ is an auxiliary type for every $j \in n$;

2 ) if $\tau$ is an auxiliary type, then $[\tau]$ is the auxiliary type;

3) if $\tau_{0}, \ldots, \tau_{k}$ are auxiliary types and $k \geq 1$, then $\left[\tau_{0}, \ldots, \tau_{k}\right]$ is an auxiliary type.

A type will be called basic if it is not auxiliary.

Thus for a second-order type $\tau \equiv\left[\tau_{0}, \ldots, \tau_{k}\right]$ the index set $k+1$ is decomposed on two subsets $M(\tau)$ and $N(\tau)$ so that for any $\mu \in M(\tau)$ the type $\tau_{\mu}$ is basic and for any $v \in N(\tau)$ the type $\tau_{\nu}$ is auxiliary.

\section{Formations and Terminals}

Further in the paper $K_{0}, \ldots, K_{n-1}$ are fixed auxiliary sets. If $n=0$ then all the fixed sets are absent.

Define the formation $G \equiv\left[P_{0}, \ldots, P_{l-1}\right]$ of the rank $l \in \omega_{0}$ in the following way:

1) $G \equiv\left[P_{0}, \ldots, P_{-1}\right] \equiv \varnothing$ for $l=0$;

2) $G \equiv\left[P_{0}, \ldots, P_{0}\right] \equiv P$ for $l=1$;

3) $G \equiv\left[P_{0}, \ldots, P_{l-1}\right] \equiv\left(P_{i} \mid i \in l\right) \equiv\left(P_{0}, \ldots, P_{l-1}\right)$ for $l \geq 2$.

Further we fix the auxiliary formation $H \equiv\left[K_{0}, \ldots, K_{n-1}\right]$ of the rank $n \in \omega_{0}$.

Define the formation $X \equiv\left[A_{0}, \ldots, A_{m}, K_{0}, \ldots, K_{n-1}\right]$ of the rank $m+1 \mid n$ over the set $H$ in the following way:

1) $X \equiv\left[A_{0}, \ldots, A_{m}, K_{0}, \ldots, K_{n-1}\right] \equiv\left[A_{0}, \ldots, A_{m}\right]$ for $n=0$ and $m \in \omega_{0}$; 
2) $X \equiv\left[A_{0}, \ldots, A_{m}, K_{0}, \ldots, K_{n-1}\right] \equiv\left\langle\left[A_{0}, \ldots, A_{m}\right],\left[K_{0}, \ldots, K_{n-1}\right]\right\rangle$ for $n \geq 1$ and $m \in \omega_{0}$.

The sets $A_{0}, \ldots, A_{m}$ are called basic in $X$. A formation $X$ may be without auxiliary sets but should contain at least one basic set.

Define the terminals $\tau(X)$ of the semitypes $\tau$ over the formation $X$ by induction:

1) $\langle i, 1\rangle(X) \equiv A_{j}$;

2) $\langle j, 0\rangle(X) \equiv K_{j}$;

3) if $\tau$ is a semitype then $[\tau](X) \equiv \mathcal{P}(\tau(X))$, where $\mathcal{P}$ denotes the operation of taking power-set of the intended set;

$4)$ if $\tau_{0}, \ldots, \tau_{k}$ are semitypes and $k \geq 1$ then $\left(\tau_{0}, \ldots, \tau_{k}\right)(X) \equiv \tau_{0}(X) \times \ldots \times \tau_{k}(X)$.

Thus for semitypes $\tau_{0}, \ldots, \tau_{k}$ with $k \geq 1$, for the type $\tau \equiv\left[\tau_{0}, \ldots, \tau_{k}\right]$, and for its semitype $\check{\tau}=\left(\tau_{0}, \ldots, \tau_{k}\right)$ the following equalities $\tau(X)=\mathcal{P}\left(\tau_{0}(X) \times \ldots \times \tau_{k}(X)\right)$ and $\check{\tau}(X)=\tau_{0}(X) \times \ldots \times \tau_{k}(X)$ are fulfilled.

\section{Signatures and Formulas}

A non-empty set $\Theta$ of types $\tau$ will be called the type domain of rank $m+1 \mid n$ if $\tau \in \Theta$ implies $p \tau \in \Theta$ for every parent $p \tau$ of the type $\tau$. In a type domain $\Theta$ select the belonging type subdomain $\Theta_{b} \equiv\left\{\tau \in \Theta \mid \exists k \in \omega_{0} \exists \tau_{0}, \ldots, \tau_{k} \in\right.$ $\left.\Theta\left(\tau=\left[\tau_{0}, \ldots, \tau_{k}\right]\right)\right\}$.

A collection $\Sigma_{c} \equiv\left(\Sigma_{c}^{\tau} \mid \tau \in \Theta\right)$ of collections $\Sigma_{c}^{\tau} \equiv\left(\sigma_{\omega}^{\tau} \mid \omega \in \Omega_{\tau}\right)$ of constants $\sigma_{\omega}^{\tau}$ of the types $\tau$ will be called the signature of constants of the type domain $\Theta$. Sets $\Omega_{\tau}$ may be empty, and then $\Sigma_{c}^{\tau}=\varnothing$.

The constants $\sigma_{\omega}^{\tau}$ of the first-order type $\tau$ are called individual or objective ones. Constants of other types are called predicate.

A collection $\Sigma_{e} \equiv\left(\delta_{\tau} \mid \tau \in \Theta\right)$ of binary predicate symbols of (generalized) equalities $\delta_{\tau}$ of the types $\tau$ will be called a signature of (generalized) equalities of the type domain $\Theta$. In follows from the definition of the type domain that for every equality symbol $\delta_{\tau}$ the collection $\Sigma_{e}$ contains necessarily the equality symbols $\delta_{p \tau}$ for every parent $p \tau$ of the type $\tau$.

A collection $\Sigma_{b} \equiv\left(\epsilon_{\tau} \mid \tau \in \Theta\right)$ of binary predicate symbols of (generalized) belongings $\epsilon_{\tau}$ of the types $\tau$ will be called the signature of (general) belongings of the type domain $\Theta$.

A collection $\Sigma_{v} \equiv\left(\Sigma_{v}^{\tau} \mid \tau \in \Theta\right)$ of denumerable sets $\Sigma_{v}^{\tau}$ of variables $x^{\tau}, y^{\tau}, \ldots$ of the type $\tau$ will be called the signature of variables of the type domain $\Theta$. The sets $\Sigma_{v}^{\tau}$ may be empty. The variables $x^{\tau}, y^{\tau}, \ldots$ of the first-order types $\tau$ are called individual or objective. The variables of other types are called predicate.

Further we shall always assume that for every type $\tau \in \Theta$ there are either constants or variables of this type.

The quadruple $\Sigma^{g} \equiv \Sigma_{c}\left|\Sigma_{e}\right| \Sigma_{b} \mid \Sigma_{v}$ will be called a (polygrade) generalized signature of the rank $m+1 \mid n$ or a signature with generalized equalities and belongings.

The language $L\left(\Sigma^{g}\right)$ of the generalized signature $\Sigma^{g}$ consists of:

1) all types $\tau$ from the type domain $\Theta$;

2) all members of all signatures from $\Sigma^{g}$;

3) logical symbols $\neg, \vee, \wedge, \Rightarrow, \forall$, and $\exists$.

4) parenthesis.

If the type domain $\Theta$ contains first- and second-order types only and at least one second-order type, then we shall say that the signature $\Sigma^{g}$ and the language $L\left(\Sigma^{g}\right)$ have the second order (see Mendelson, 1997, Appendix; van Dalen, 1983, p. 4). In this case the notations $\Sigma_{2}^{g}$ and $L\left(\Sigma_{2}^{g}\right)$ will be used.

Constants and variables of a type $\tau$ are called terms of the type $\tau$ of the language $L\left(\Sigma^{g}\right)$.

The atomic formulas of the language $L\left(\Sigma^{g}\right)$ are defined in the following way:

1) if $q$ and $r$ are terms of a type $\tau \in \Theta$, then $q \delta_{\tau} r$ is an atomic formula;

2) if $\tau_{0}, \ldots, \tau_{k}$ are the types from $\Theta$ for $k \geq 0, \tau \equiv\left[\tau_{0}, \ldots, \tau_{k}\right] \in \Theta, q_{0}^{\tau_{0}}, \ldots, q_{k}^{\tau_{k}}$ are terms of types $\tau_{0}, \ldots, \tau_{k}$, respectively, and $r^{\tau}$ is a term of the type $\tau$, then $\left(q_{0}^{\tau_{0}}, \ldots, q_{k}^{\tau_{k}}\right) \epsilon_{\tau} r^{\tau}$ is an atomic formula; in particular for $k=0$ the symbol-string $q_{0}^{\tau_{0}} \epsilon_{\left[\tau_{0}\right]} r^{\left[\tau_{0}\right]}$ is an atomic formula. 
The formulas of the language $L\left(\Sigma^{g}\right)$ are constructed from atomic ones with the use of connectives $\vee, \wedge, \neg, \Rightarrow$, quantifiers $\exists x^{\tau}$ and $\forall x^{\tau}$ with respect to variables $x^{\tau}$, and parenthesis.

The logical axiom schemes of (polygrade) type theory in the language $L\left(\Sigma^{g}\right)$ of the generalized signature $\Sigma^{g}$ are schemes of the predicate calculus where variables and terms substituting each other must be of the same type $\tau \in \Omega$.

In addition to these axiom schemes consider the following equality axioms for types $\tau \in \Theta$ :

E1) $\forall x^{\tau}\left(x \delta_{\tau} x\right)$;

E2) $\forall x^{\tau}, y^{\tau}\left(x \delta_{\tau} y \Rightarrow y \delta_{\tau} x\right)$;

E3) $\forall x^{\tau}, y^{\tau}, z^{\tau}\left(x \delta_{\tau} y \wedge y \delta_{\tau} z \Rightarrow x \delta_{\tau} z\right)$;

E4) $\left.\forall x_{0}^{\tau_{0}}, y_{0}^{\tau_{0}}, \ldots, x_{k}^{\tau_{k}}, y_{k}^{\tau_{k}}, u^{\tau}, v^{\tau}\left(x_{0} \delta_{\tau_{0}} y_{0} \wedge \ldots \wedge x_{k} \delta_{\tau_{k}} y_{k} \wedge u \delta_{\tau} v \Rightarrow\left(\left(x_{0}, \ldots, x_{k}\right) \epsilon_{\tau} u \Leftrightarrow\left(y_{0}, \ldots, y_{k}\right) \epsilon_{\tau} v\right)\right)\right)$, where $\tau \equiv$ $\left[\tau_{0}, \ldots, \tau_{k}\right]$.

Axiom E4 is the initial principle of change of equals.

The inference rules in the depicted type theory are:

$$
\frac{\varphi, \varphi \Rightarrow \psi}{\psi}(M P) \quad \text { and } \quad \frac{\varphi\left(x^{\tau}\right)}{\forall x^{\tau} \varphi\left(x^{\tau}\right)} \quad(\text { Gen }) .
$$

If there are nonlogical axioms or axiom schemes written by second-order formulas, we shall say that a (mathematical) generalized second-order theory is given.

\section{Mathematical Systems of the Signature $\Sigma^{g}$ With Generalized Equalities and Belongings}

\subsection{Definition of a Mathematical System of the Generalized Signature $\mathbf{\Sigma}^{g}$}

Let $\Sigma^{g}$ be a fixed signature of the rank $m+1 \mid n$ defined in the previous section. Fix also a formation $X \equiv$ $\left[A_{0}, \ldots, A_{m}, K_{0}, \ldots, K_{n-1}\right]$ of the rank $m+1 \mid n$.

For the formation $X$ and the signature $\Sigma^{g}$ consider the following collections:

1) the collection $S_{c} \equiv\left(S_{c}^{\tau} \mid \tau \in \Theta\right)$ of collections $S_{c}^{\tau} \equiv\left(s_{\omega}^{\tau} \mid \omega \in \Omega_{\tau}\right)$ of constant structures $s_{\omega}^{\tau} \in \tau(X)$ of the types $\tau$

2) the collection $S_{e} \equiv\left(\approx_{\tau} \mid \tau \in \Theta\right)$ of generalized equality relations $\approx_{\tau} \subset \tau(X) \times \tau(X)$ of the types $\tau$ on the sets $\tau(X)$, containing the usual set-theoretic equality relations $=$ on the sets $\tau(X)$, i.e., such relations $\approx_{\tau}$ that for every elements $r, s \in \tau(X)$ the equality $r=s$ implies the generalized equality $r \approx_{\tau} s$;

3) the collection $S_{b} \equiv\left(\leftarrow_{\tau} \mid \tau \in \Theta_{b}\right)$ of generalized belonging correspondences $\varangle_{\tau} \subset \check{\tau}(X) \times \tau(X)$ of the types $\tau$, containing the usual set-theoretic belonging correspondences $\in$ from the sets $\check{\tau}(X)$ into the sets $\tau(X)$, i.e., such correspondences $<_{\tau}$ that for every elements $p \in \check{\tau}$ and $P \in \tau(X)$ the belonging $p \in P$ implies the generalized belonging $p<_{\tau} P$;

4) the collection $S_{v} \equiv(\tau(X) \mid \tau \in \Theta)$ of the terminals $\tau(X)$ of the types $\tau$ over the formation $X$.

The quadruple $S \equiv\left(S_{c}, S_{e}, S_{b}, S_{v}\right)$ of the above-mentioned collections will be called a (polygrade) superstructure of the signature $\Sigma^{g}$ over the formation $X$.

The pair $U \equiv(X, S)$ will be called a mathematical system of the generalized signature $\Sigma^{g}$ with the support $X$ and the superstructure $S$. This notion is a generalization of the notion of an algebraic system of the signature $\Sigma_{1}$ (see Ershov \& Palyutin, 1984, §15).

The mathematical system $U \equiv(X, S)$ will be called also an interpretation of the signature $\Sigma^{g}$ on the support $X$.

Further for a type $\tau=\left[\tau_{0}, \ldots, \tau_{k}\right]$ and elements $p \equiv(p(0), \ldots, p(k)), q \equiv(q(0), \ldots, q(k)) \in \check{\tau}(X)=\tau_{0}(X) \times \cdots \times$ $\tau_{k}(X)$ along with $p(0) \approx_{\tau_{0}} q(0) \wedge \cdots \wedge p(k) \approx_{\tau_{k}} q(k)$ we shall also write $p \approx_{\check{\tau}} q$.

\subsection{Concordance of Mathematical Systems of the Generalized Second-Order Signature}

Two mathematical systems $U \equiv(X, S)$ and $V \equiv(Y, T)$ of the signature $\Sigma_{2}^{g}$ will be called $H$-concordant if:

1) for every auxiliary type $\tau \in \Theta$ and every $\omega \in \Omega_{\tau}$ the constants $s_{\omega}^{\tau} \in \tau(X)$ and $t_{\omega}^{\tau} \in \tau(Y)=\tau(X)$ coincide, where by definition of terminals $\tau(X)=\tau(Y)$; 
2) for every auxiliary type $\tau \in \Theta$ the equalities $\approx_{\tau} \subset \tau(X) \times \tau(X)$ and $\approx_{\tau} \subset \tau(Y) \times \tau(Y)$ coincide, where as above $\tau(Y) \times \tau(Y)=\tau(X) \times \tau(X)$

3) for every auxiliary type $\tau \in \Theta_{b}$ the belongings $<_{\tau} \subset \check{\tau}(X) \times \tau(X)$ and $\leftarrow_{\tau} \subset \check{\tau}(Y) \times \tau(Y)$ coincide, where by the same reason $\check{\tau}(Y) \times \tau(Y)=\tau(\check{X}) \times \tau(X)$;

4) for every suite $p \equiv(p(0), \ldots, p(k)) \in s_{\omega}^{\tau} \subset \check{\tau}(X)=\tau_{0}(X) \times \cdots \times \tau_{k}(X)$ there exists a suite $q \equiv(q(0), \ldots, q(k)) \in$ $t_{\omega}^{\tau} \subset \check{\tau}(Y)=\tau_{0}(Y) \times \cdots \times \tau_{k}(Y)$ such that $q(v)=p(v)$, and for every $q$ there exists $p$ such that $p(v)=q(v)$ for every $v \in N(\tau)$ and every type $\tau \equiv\left[\tau_{0}, \ldots, \tau_{k}\right]$ such that $M(\tau) \neq \varnothing$ and $N(\tau) \neq \varnothing$.

The property of $H$-concordance means the identity of the systems $U$ and $V$ with respect to all elements connected with the auxiliary set $H$.

The generalized equalities $\approx_{\tau}$ and the generalized belongings $<_{\tau}$ admit some additional conditions.

A system $U$ will be called balanced, if $\forall P, Q \in \tau(X)\left(P \approx_{\tau} Q \Leftrightarrow \forall p \in P \exists q \in Q\left(q \approx_{\breve{\tau}} p\right) \wedge \forall q \in Q \exists p \in P\left(p \approx_{\tau} q\right)\right)$, where $\tau_{0}, \ldots, \tau_{k} \in \Theta, k \geq 0$ and $\tau \equiv\left[\tau_{0}, \ldots, \tau_{k}\right] \in \Theta$.

A system $U$ will be called regular, if $\forall p \in \breve{\tau}(X) \forall P \in \tau(X)\left(p \leftarrow_{\tau} P \Leftrightarrow \exists q \in P\left(p \approx_{\breve{\tau}} q\right)\right)$, where $\tau_{0}, \ldots, \tau_{k} \in \Theta, k \geq 0$ and $\tau \equiv\left[\tau_{0}, \ldots, \tau_{k}\right] \in \Theta$.

A system $U$ will be called normal, if $\forall p, q \in \sigma(X)\left(p \approx_{\sigma} q \Leftrightarrow p=q\right) \wedge \forall p \in \breve{\tau}(X) \forall P \in \tau(X)\left(p<_{\tau} P \Leftrightarrow p \in P\right)$.

A system $U$ will be called extensional, if $\forall P, Q \in \tau(X)\left(P \approx_{\tau} Q \Leftrightarrow \forall p\left(p<_{\tau} P \Rightarrow p<_{\tau} Q\right) \wedge \forall q\left(q<_{\tau} Q \Rightarrow q<_{\tau} P\right)\right)$, where $\tau \in \Theta_{b}$.

\subsection{Evaluations and Models}

An evaluation on a system $U \equiv(X, S)$ of the signature $\Sigma^{g}$ is a mapping $\gamma$, defined on the set of all variables of the signature $\Sigma^{g}$ and associating with the variable $x^{\tau}$ of the type $\tau \in \Theta$ the element $\gamma\left(x^{\tau}\right)$ of the terminal $\tau(X)$ (see Ershov \& Palyutin, 1984, §16; Takeuti, 1975, 16.17]). The pair $(U, \gamma)$ consisting of the system $U$ of the signature $\Sigma^{g}$ and and the evaluation $\gamma$ on $U$ will be called an evaluated mathematical system of the signature $\Sigma^{g}$.

Evaluated mathematical systems $(U, \gamma)$ and $(V, \delta)$ of the signature $\Sigma^{g}$ will be called $H$-concordant if:

1) the systems $U$ and $V$ are $H$-concordant;

2) for every auxiliary type $\tau \in \Theta$ the evaluations $\gamma\left(x^{\tau}\right) \in \tau(X)$ and $\delta\left(x^{\tau}\right) \in \tau(Y)=\tau(X)$ coincide i.e., $\gamma\left(x^{\tau}\right)=\delta\left(x^{\tau}\right)$ (see 4.1);

3) for every suite $p \equiv(p(0), \ldots, p(k)) \in \gamma\left(x^{\tau}\right) \subset \breve{\tau}(X)=\tau_{0}(X) \times \cdots \times \tau_{k}(X)$ there exists a suite $q \equiv(q(0), \ldots, q(k)) \in$ $\delta\left(x^{\tau}\right) \subset \breve{\tau}(Y)=\tau_{0}(Y) \times \cdots \times \tau_{k}(Y)$ such that $q(v)=p(v)$ and, for every $q$ there exists $p$ such that $p(v)=q(v)$ for every $v \in N(\tau)$ and every type $\tau=\left[\tau_{0}, \ldots, \tau_{k}\right]$ such that $M(\tau) \neq \varnothing$ and $N(\tau) \neq \varnothing$.

The property of $H$-concordance means the identity of the evaluated systems $(U, \gamma)$ and $(V, \delta)$ with respect to all elements connected with the auxiliary set $H$.

An evaluation $\gamma$ on a system $U$ and an evaluation $\delta$ on a system $V$ will be called $H$-concordant if they satisfy conditions 2) and 3) from the previous definition.

Define the value $q[\gamma]$ of a term $q$ relatively the evaluation $\gamma$ on the system $U$ in the following way (see Ershov \& Palyutin, 1984, §16; Mal'cev, 1970, §6; Mendelson, 1997, 2.2; Schoenfield, 1967, 3.2):

1) if $\sigma^{\tau}$ is a constant of a type $\tau \in \Theta$, then $\sigma^{\tau}[\gamma] \equiv s^{\tau}$;

2) if $x^{\tau}$ is a variable of a type $\tau \in \Theta$, then $x^{\tau}[\gamma] \equiv \gamma\left(x^{\tau}\right)$.

Define the satisfaction of a formula $\varphi$ of the language $L\left(\Sigma_{2}^{q}\right)$ on a system $U$ of the signature $\Sigma_{2}^{q}$ relatively an evaluation $\gamma$ (in designation $U \models \varphi[\gamma]$ ) by induction in the following way (see Mendelson, 1997, 2.2; Schoenfield, 1967, 3.2; Takeuti, 1975, 16.17):

1) if $q$ and $r$ are terms of a type $\tau \in \Theta$ and $\varphi \equiv\left(q \delta_{\tau} r\right)$, then $U \vDash \varphi[\gamma]$ is equivalent to $q[\gamma] \approx_{\tau} r[\gamma]$;

2) if $\tau_{0}, \ldots, \tau_{k}$ are types from $\Theta$ for $k \geq 0, \tau \equiv\left[\tau_{0}, \ldots, \tau_{k}\right] \in \Theta, q_{0}, \ldots, q_{k}$ are terms of the types $\tau_{0}, \ldots, \tau_{k}$, respectively, $r$ is a term of the type $\tau$, and $\varphi \equiv\left(q_{0}, \ldots, q_{k}\right) \epsilon_{\tau} r$, then $U \models \varphi[\gamma]$ is equivalent to $\left(q_{0}[\gamma], \ldots, q_{k}[\gamma]\right)<_{\tau} r[\gamma]$;

3) if $\varphi \equiv \neg \psi$, then $U \vDash \varphi[\gamma]$ iff $U \vDash \psi[\gamma]$ is not true;

4) if $\varphi \equiv(\psi \vee \xi)$, then $U \vDash \varphi[\gamma]$ iff $U \vDash \psi[\gamma]$ or $U \vDash \xi[\gamma]$; 
5) if $\varphi \equiv(\psi \wedge \xi)$, then $U \vDash \varphi[\gamma]$ iff $U \vDash \psi[\gamma]$ and $U \vDash \xi[\gamma]$;

6) if $\varphi \equiv(\psi \Rightarrow \xi)$, then $U \vDash \varphi[\gamma]$ iff that $U \vDash \psi[\gamma]$ implies $U \vDash \xi[\gamma]$;

7) if $\varphi \equiv \exists x^{\tau} \psi$, then $U \vDash \varphi[\gamma]$ is equivalent to $U \models \psi\left[\gamma^{\prime}\right]$ for some evaluation $\gamma^{\prime} H$-concordant with $\gamma$ and such that $\gamma^{\prime}\left(y^{\sigma}\right)=\gamma\left(y^{\sigma}\right)$ for every variable $y^{\sigma} \neq x^{\tau}$;

8) if $\varphi \equiv \forall x^{\tau} \psi$, then $U \vDash \varphi[\gamma]$ is equivalent to $U \models \psi\left[\gamma^{\prime}\right]$ for every evaluation $\gamma^{\prime} H$-concordant with $\gamma$ and such that $\gamma^{\prime}\left(y^{\sigma}\right)=\gamma\left(y^{\sigma}\right)$ for every variable $y^{\sigma} \neq x^{\tau}$.

Note that bringing into use in points 7) and 8) of this definition the additional (in comparison with Ershov \& Palyutin, 1984, §16; Mendelson, 1997, 2.2; Shoenfield, 1967, 3.2) property of $H$-concordance of the evaluations $\gamma$ and $\gamma^{\prime}$ is stipulated by the initial polygrade structure of considered mathematical systems and by the presence of the fixed auxiliary formation $H \equiv\left[K_{0}, \ldots, K_{n-1}\right]$.

Let $\Phi$ be a set of formulas of the language $L\left(\Sigma_{2}^{q}\right)$. An evaluated mathematical system $(U, \gamma)$ of the signature $\Sigma_{2}^{q}$ will be called a model for the set $\Phi$ if $U \models \varphi[\gamma]$ for every formula $\varphi \in \Phi$ (see Ershov \& Palyutin, 1984, §17).

A model $(U, \gamma)$ for $\Phi$ will be called a model of the second order if at least one formula from $\Phi$ contains at least one second order variable.

A model $(U, \gamma)$ will be called balanced, regular, normal, extensional etc., if the system $U$ is the same.

A model $(U, \gamma)$ for a set $\Phi$ will be called second-order, if at least one formula from $\Phi$ contains at least one secondorder variable.

Remark that if a system $U \equiv[X, S]$ is considered in an axiomatic set theory, then the satisfaction of a closed formula $\varphi$ of the language $L\left(\Sigma_{2}^{g}\right)$ under any evaluation $\gamma$ is reduced to correctness of the relativization $\varphi^{r}$ of $\varphi$ on the corresponding terminal of the support $X$ in this set theory.

In particular, since equality axioms $E 1-E 4$ are closed formulas, their relativizations $E 1^{r}-E 4^{r}$ take the following forms:

$\left.E 1^{r}\right) \quad \forall x \in \tau(X)\left(x \approx_{\tau} x\right)$

$\left.E 2^{r}\right) \quad \forall x, y \in \tau(X)\left(x \approx_{\tau} y \Rightarrow y \approx_{\tau} x\right)$

$\left.E 3^{r}\right) \quad \forall x, y, z \in \tau(X)\left(x \approx_{\tau} y \wedge y \approx_{\tau} z \Rightarrow x \approx_{\tau} z\right)$;

$\left.E 4^{r}\right) \quad \forall x_{0}, y_{0} \in \tau_{0}(X) \ldots \forall x_{k}, y_{k} \in \tau_{k}(X) \forall u, v \in \tau(X)\left(x_{0} \approx_{\tau_{0}} \wedge \ldots \wedge x_{k} \approx_{\tau_{k}} y_{k} \wedge u \approx_{\tau} v \Rightarrow\left(\left(x_{0}, \ldots, x_{k}\right) \leftarrow_{\tau} u \Leftrightarrow\right.\right.$ $\left.\left.\left(y_{0}, \ldots, y_{k}\right)<_{\tau} v\right)\right)$, where $\tau \equiv\left[\tau_{0}, \ldots, \tau_{k}\right], k \geq 0$ and all types are in $\Theta$.

The satisfaction of formulas $E 1^{r}-E 3^{r}$ means that all generalized equalities $\approx_{\tau}$ are equivalence relations on corresponding sets $\tau(X)$, and the satisfaction of formula $E 4^{r}$ means the property of replacing of equals in the atomic formula with the generalized belonging $<_{\tau}$.

Further we shall say that a system $U$ of the signature $\Sigma_{2}^{g}$ has true generalized equalities and belongings, if axioms $E 1-E 4$ from section 3 are satisfied on $U$ with respect to some (and consequently to any) evaluation $\gamma$. It means that formulas $E 1^{r}-E 4^{r}$ are correct for the system $U$ in the used set theory.

\subsection{The Generalized Equality of Values of Evaluations and Satisfiability}

For every formula $\varphi$ of the language $L\left(\Sigma_{2}^{q}\right)$ we define the formula $\varphi^{*}$ by means of induction:

1) $\varphi^{*} \equiv \varphi$ for every atomic formula $\varphi$;

2) $(\psi \wedge \xi)^{*} \equiv \psi^{*} \wedge \xi^{*}$

3) $(\neg \psi)^{*} \equiv \neg \psi^{*}$;

4) $\left(\exists x^{\tau} \psi\right)^{*} \equiv \exists x^{\tau} \psi^{*}$;

5) $(\psi \vee \xi)^{*} \equiv \neg\left(\neg \psi^{*} \wedge \neg \xi^{*}\right)$;

6) $(\psi \Rightarrow \xi)^{*} \equiv \neg\left(\psi^{*} \wedge \neg \xi^{*}\right)$;

7) $\left(\forall x^{\tau} \psi\right)^{*} \equiv \neg\left(\exists x^{\tau}\left(\neg \psi^{*}\right)\right)$.

Say that a formula $\varphi$ is normalizable, if for every mathematical $\Sigma_{2}^{q}$-system $U$ and every evaluation $\gamma$ on $U$ the following condition holds: $U \models \varphi[\gamma] \Leftrightarrow U \models \varphi^{*}[\gamma]$. 
Lemma 1 Let formulas $\psi$ and $\xi$ be normalizable. Then formulas $\psi \wedge \xi, \neg \psi, \psi \vee \xi, \psi \Rightarrow \xi, \forall x^{\tau} \psi$ and $\exists x^{\tau} \psi$ are normalizable too.

A proof of this lemma uses the definition of satisfiability and some well known tautologies only, so it is omitted.

Proposition 1 Every formula of the language $L\left(\Sigma_{2}^{g}\right)$ of the generalized second-order signature $\Sigma_{2}^{g}$ is normalizable.

Proof. Denote by $\Phi$ the set of all formulas of the language $L\left(\Sigma_{2}^{g}\right)$. The subset of the set $\Phi$ consisting o formulas containing at most $n \in \omega_{0}$ logical symbols $\neg, \wedge, \Rightarrow, \vee, \exists, \forall$, denote by $\Phi_{n}$. It is clear that $\Phi=\bigcup\left(\Phi_{n} \mid n \in \omega_{0}\right)$.

Prove by the complete induction principle the following assertion $A(n)$ : every formula $\varphi \in \Phi$ is normalizable.

If $n=0$, then the formula $\varphi$ is atomic, and so by the definition, of the operation $\varphi \mapsto \varphi^{*}$ we have $\varphi^{*} \equiv \varphi$. Consequently the assertion $A(0)$ is true.

Suppose for all $m<n$ the assertion $A(m)$ is true. Let $\varphi \in \Phi_{n}$. If $\varphi \equiv \psi \wedge \xi, \varphi \equiv \neg \psi, \varphi \equiv \exists x^{\tau} \psi, \varphi \equiv \psi \vee \xi, \varphi \equiv \psi \Rightarrow \xi$ or $\varphi \equiv \forall x^{\tau} \psi$, then $\psi, \xi \in \Phi_{n-1}$. Therefore by the induction hypothesis the formulas $\psi$ and $\xi$ are normalizable. By the previous Lemma 1 the formula $\varphi$ is normalizable. Hence the assertion $A(n)$ is true.

Proposition 2 Let $U$ be a mathematical system of the second-order signature $\Sigma_{2}^{g}$ with true generalized equalities and belongings. Then for every formula $\varphi$ of the language $L\left(\Sigma_{2}^{g}\right)$ and every $H$-concordant evaluations $\gamma$ and $\delta$ on the system $U$ such that $\gamma\left(x^{\tau}\right) \approx_{\tau} \delta\left(x^{\tau}\right)$ for every variable $x^{\tau}$ of every type $\tau \in \Theta$ the properties $U \models \varphi[\gamma]$ and $U \models \varphi[\delta]$ are equivalent.

Proof. The set of all formulas $\varphi$ of the language $L\left(\Sigma_{2}^{g}\right)$ constructed by induction from the atomic formulas with the use of connectives $\neg$ and $\wedge$ and quantifier $\exists$ denote by $\Psi$. The subset of the set $\Psi$ consisting of formulas containing at most $n \in \omega_{0}$ logical symbols $\neg, \wedge$, and $\exists$ denote by $\Psi_{n}$. It is clear that $\Psi=\bigcup\left(\Psi_{n} \mid n \in \omega_{0}\right)$.

Prove by the complete induction principle the assertion of Proposition 2 A(n): for every formula $\varphi \in \Psi_{n}$ and every mentioned evaluations $\gamma$ and $\delta$ Proposition 2 is true.

Let $n=0$ and $\varphi \in \Psi_{0}$. Then $\varphi$ is an atomic formula. At first consider the atomic formula $\varphi$ of the form $q^{\tau} \delta_{\tau} r^{\tau}$. Suppose that $q^{\tau}=x^{\tau}$ and $r^{\tau}=\sigma_{\omega}^{\tau}$. Then $U \models \varphi[\gamma]$ is equivalent to $\gamma(x) \approx_{\tau} s_{\omega}^{\tau}$ and $U \models \varphi[\delta]$ is equivalent to $\delta(x) \approx_{\tau} s_{\omega}^{\tau}$. Since, by our condition $\gamma(x) \approx_{\tau} \delta(x)$, assuming $U \vDash \varphi[\gamma]$ and using axioms $E 2^{r}$ and $E 3^{r}$ we infer $U \vDash \varphi[\delta]$. The inverse inference is checked in the same way. For the terms $q^{\tau}$ and $r^{\tau}$ of other forms the reasons are quite similar.

Now consider the atomic formula $\varphi$ of the form $\left(q_{0}^{\tau_{0}}, \ldots, q_{k}^{\tau_{k}}\right) \varepsilon_{\tau} r^{k}$ for $\tau \equiv\left[\tau_{0}, \ldots, \tau_{k}\right] \in \Theta_{b}$. Assume that $q_{\lambda}^{\tau_{\lambda}}=x_{\lambda}^{\tau_{\lambda}}$ and $r^{\tau}=u^{\tau}$ for some variables $x_{\lambda}$ and $u$. Then $U \models \varphi[\gamma]$ is equivalent to $\left(\gamma\left(x_{0}\right), \ldots, \gamma\left(x_{k}\right)\right)<_{\tau} \gamma(u)$ and $U \models \varphi[\delta]$ is equivalent to $\left(\delta\left(x_{0}\right), \ldots, \delta\left(x_{k}\right)\right)<_{\tau} \delta(u)$.

Suppose $U \vDash \varphi[\gamma]$. Since, by our condition, $\gamma\left(x_{\lambda}^{\tau_{\lambda}}\right) \approx_{\tau_{\lambda}} \delta\left(x_{\lambda}^{\tau_{\lambda}}\right)$, assuming $U \models \varphi[\gamma]$ and using axiom $E 4^{r}$ we infer $U \vDash \varphi[\delta]$. The inverse inference is checked in the same way. For the terms $q_{\lambda}^{\tau_{\lambda}}$ and $r^{\tau}$ of other kinds the reasons are quite similar.

Assume that assertion $A(m)$ is true for every $m<n$. Let $\varphi \equiv \exists x^{\tau} \psi$. Then $\psi \in \Psi_{n-1}$. Let be given some $H$ concordant evaluations $\gamma$ and $\delta$ such that $\gamma\left(x^{\tau}\right) \approx_{\tau} \delta\left(x^{\tau}\right)$.

Suppose $U \vDash \varphi[\gamma]$. It is equivalent to $U \vDash \psi\left[\gamma^{\prime}\right]$ for some evaluation $\gamma^{\prime}, H$-concordant with $\gamma$ and such that $\gamma^{\prime}(y)=\gamma(y)$ for any $y^{\sigma} \neq x^{\tau}$.

Define an evaluation $\delta^{\prime}$ on $U$ setting $\delta^{\prime}(y) \equiv \delta(y)$ for every $y^{\sigma} \neq x^{\tau}$ and $\delta^{\prime}(x) \equiv \gamma^{\prime}(x)$. Then $\delta^{\prime}(y)=\delta(y) \approx_{\sigma} \gamma(y)=$ $\gamma^{\prime}(y)$ and $\delta^{\prime}(x)=\gamma^{\prime}(x)$, i.e., $\delta^{\prime}(x) \approx_{\tau} \gamma^{\prime}(x)$.

Check that the evaluations $\delta^{\prime}$ and $\gamma^{\prime}$ are $H$-concordant. If $\sigma$ is an auxiliary first-order type, then $\delta^{\prime}\left(y^{\sigma}\right) \equiv \delta(y)=$ $\gamma(y)=\gamma^{\prime}(y)$. If $\tau$ is an auxiliary first-order type, then $\delta^{\prime}\left(x^{\tau}\right)=\gamma^{\prime}\left(x^{\tau}\right)$.

Let $\sigma$ and $\tau$ be second-order types. Let $p \in \delta^{\prime}\left(y^{\sigma}\right)=\delta(y)$. Since $\delta$ and $\gamma$ are $H$-concordant, for $p$ there exists $q \in \gamma(y)$ such that $q(v)=p(v)$ for every $v \in N(\sigma)$. Since $\gamma$ and $\gamma^{\prime}$ are $H$-concordant, there exists $r \in \gamma^{\prime}(y)$ such that $r(v)=q(v)$. So for $p$ there is $r \in \gamma^{\prime}(y)$ such that $r(v)=p(v)$ for every $v \in N(\sigma)$. The inverse property can be established in the same way. , The property of $H$-concordancy for $x^{\tau}$ holds automatically because $\delta^{\prime}\left(x^{\tau}\right) \equiv \gamma^{\prime}\left(x^{\tau}\right)$.

Since $\delta^{\prime}$ and $\gamma^{\prime}$ are $H$-concordant and $\delta^{\prime}\left(x^{\tau}\right) \approx \gamma^{\prime}\left(x^{\tau}\right)$ then by our condition, $U \models \psi\left[\gamma^{\prime}\right] \leftrightarrow U \models \psi\left[\delta^{\prime}\right]$. Consequently we obtain the property $U \models \psi\left[\delta^{\prime}\right]$. By construction, $\delta^{\prime}(y)=\delta(y)$ for every $y^{\sigma} \neq x^{\tau}$.

Check that the evaluations $\delta$ and $\delta^{\prime}$ are $H$-concordant. If $\sigma$ is an auxiliary first-order type, then $\delta^{\prime}\left(y^{\sigma}\right)=\delta(y)$. If $\tau$ 
is an auxiliary first-order type, then $\delta^{\prime}\left(x^{\tau}\right)=\gamma^{\prime}(x)=\gamma(x)=\delta(x)$.

Let $\sigma$ and $\tau$ bee second-order types. Since $\delta^{\prime}\left(y^{\sigma}\right)=\delta(y)$, the property of $H$-concordancy obviously holds. Let $p \in \delta(x)$. Since $\delta$ and $\gamma$ are $H$-concordant, we see that there exists $q \in \gamma(x)$ such that $q(v)=p(v)$. Since $\gamma$ and $\gamma^{\prime}$ are $H$-concordant, there exists $r \in \gamma^{\prime}(x)=\delta^{\prime}(x)$ such that $r(v)=q(v)$. So $r(v)=p(v)$ for every $v \in N(\tau)$. The inverse property is established in the same way.

By the definition of satisfiability, we conclude that $U \models \varphi[\delta]$. The inverse inference of $U \models \varphi[\gamma]$ from $U \models \varphi[\delta]$ is established quite analogously.

Now let $\varphi \equiv \psi \wedge \xi$. Then $\psi, \xi \in \Psi_{n-1}$. Consequently, $U \vDash \psi[\gamma] \leftrightarrow U \vDash \psi[\delta]$ and $U \vDash \xi[\gamma] \leftrightarrow U \vDash \xi[\delta]$. From here $(U \models \psi[\gamma] \& U \models \xi[\gamma]) \leftrightarrow(U \models \psi[\delta] \& U \models \xi[\delta])$. So $U \models \varphi[\gamma] \leftrightarrow U \models \varphi[\delta]$.

Finally, let $\varphi \equiv \neg \psi$. Then $\psi \in \Psi_{n-1}$. Consequently, $U \vDash \psi[\gamma] \leftrightarrow U \vDash \psi[\delta]$. From here $U \vDash \varphi[\gamma] \leftrightarrow \neg(U \vDash$ $\psi[\gamma]) \leftrightarrow \neg(U \models \psi[\delta]) \leftrightarrow U \models \varphi[\delta]$.

This proves that the assertion $A(n)$ is true. By the complete induction principle, the assertion $A(n)$ is true for every natural number $n \in \omega_{0}$, i.e., the assertion of Proposition 2 holds for every formula $\varphi \in \Psi$.

Now let $\varphi$ be an arbitrary formula of the language $L\left(\Sigma_{2}^{g}\right)$. In virtue of Proposition 1 we have $U \models \varphi[\gamma] \leftrightarrow U \vDash \varphi^{*}[\gamma]$ and $U \vDash \varphi[\delta] \leftrightarrow U \vDash \varphi^{*}[\delta]$. By the definition of the operation $\varphi \mapsto \varphi^{*}$, we have $\varphi^{*} \in \Psi$. As proved above $U \vDash \varphi^{*}[\gamma] \leftrightarrow U \vDash \varphi^{*}[\delta]$. In result, we obtain the equivalence $U \models \varphi[\gamma] \leftrightarrow U \vDash \varphi[\delta]$.

\subsection{An Example of a Good Model for the Second-Order Equality Axioms}

Construct for axioms $E 1-E 4$ a regular, balanced, extensional, second-order model.

Take $m=0, n=0, \rho \equiv\langle 0,1\rangle, \sigma \equiv[\rho], \Theta \equiv\{\rho, \sigma\}, \Omega_{\rho}=\varnothing, \Omega_{\sigma}=\varnothing, \Sigma_{c}^{\rho}=\varnothing$, and $\Sigma_{c}^{\sigma}=\varnothing$. Then $\Sigma_{e} \equiv\left(\delta_{\rho}, \delta_{\sigma}\right)$, $\Theta_{b}=\{\sigma\}, \Sigma_{b} \equiv\left(\varepsilon_{\tau} \mid \tau \in \Theta_{b}\right)$, i.e., $\Sigma_{b}$ consists of the symbol $\varepsilon_{\sigma}=\varepsilon_{[\rho]}$ only, and the collection $\Sigma_{v} \equiv\left(\Sigma_{v}^{\tau} \mid \tau \in \Theta\right)$ consists of a denumerable set $\Sigma_{v}^{\rho}$ of variables $x^{\rho}, y^{\rho}, \ldots$ of the first-order type $\rho$ and a denumerable set $\Sigma_{v}^{\sigma}$ of variables $u^{\sigma}, v^{\sigma}, \ldots$ of the second-order type $\sigma$.

Consider the one grade signature $\Sigma_{0} \equiv \Sigma_{c}\left|\Sigma_{e}\right| \Sigma_{b} \mid \Sigma_{v}$ of the rank 1|0, and its language $L\left(\Sigma_{0}\right)$. This language contains three atomic formulas: $x^{\rho} \delta_{\rho} y^{\rho}, u^{\sigma} \delta_{\sigma} v^{\sigma}$ and $x^{\rho} \varepsilon_{\sigma} u^{\sigma}$.

Take the set of all closed segments of straight lines on the plane as a set $A \equiv A_{0}$. Then $X=A$. Since $\Omega_{\rho}=\Omega_{\sigma}=\varnothing$, there are no constants. For segments $p, q \in A$ put $p \approx \rho q$, if $q$ is obtained from $p$ by some parallel transfer. For sets $P, Q \in \mathcal{P}(A)$ of segments put $P \approx_{\sigma} Q$, if $\left(\forall p \in P \exists q \in Q\left(p \approx_{\rho} q\right)\right) \wedge\left(\forall q \in Q \exists p \in P\left(q \approx_{\rho} p\right)\right)$. For a segment $p \in A$ and a set of segments $P \in \mathcal{P}(A)$ put $p \leftarrow_{\sigma} P$, if and only if $\exists q \in A\left(q \approx_{\rho} p \wedge q \in P\right)$, i.e., the segment $p$ can be transferred into the set $P$ with some parallel transfer.

The collection of terminals $S_{v} \equiv(\tau(X) \mid \tau \in \Theta)$ consists of the terminal $\rho(X)=A$ and the terminal $\sigma(X)=\mathcal{P}(A)$.

The constructed collections form the one grade superstructure $S$ over the set $X=A$. Consider the mathematical system $U \equiv[A, S]$ of the signature $\Sigma_{0}$.

Proposition 3 The above-constructed mathematical system $U$ together with any evaluation $\gamma$ of variables of the language $L\left(\Sigma_{0}\right)$ on the system $U$ form the regular, balanced, extensional, second-order model for equality axioms $E 1-E 4$.

Proof. The correctness of the equality axioms follows from the definition. The same is true for the balance property.

Check the extensionality property. Assume $p \in P$. Then $p \leftarrow_{\sigma} P$. Suppose the right side of the extensionality formula. By condition we conclude $p<_{\sigma} Q$. By the regularity property there exists an element $q \in Q$ such that $q \approx_{\rho} p$. The inverse finding of an element $p \in P$ for a given element $q \in Q$ such that $p \approx_{\rho} q$ is established quite similarly. In accordance with the definition of the equality $\approx_{\sigma}$ we conclude that $P \approx_{\sigma} Q$. So, we have inferred the left side of the extensionality formula. It follows from the correctness of axiom $E 4^{r}$ that the left side implies the right one.

\section{Infraproducts of Collections of Evaluated Mathematical Systems of the Generalized Second-Order Sig- nature $\Sigma_{2}^{\mathrm{g}}$}

Let $\left(U_{f} \mid f \in F\right.$ ) be a pairwise $H$-concordant collection of mathematical systems of the second-order signature $\Sigma_{2}^{g}$ with true generalized equalities and belongings.

By definition, $U_{f} \equiv\left(X_{f}, S_{f}\right)$, where $X_{f} \equiv\left[A_{0 f}, \ldots, A_{m f}, K_{0}, \ldots, K_{n-1}\right]$. 
Consider the sets $A_{i} \equiv \prod\left(A_{i f} \mid f \in F\right)$ and the formation $X \equiv\left[A_{0}, \ldots, A_{m}, K_{0}, \ldots, K_{n-1}\right] \equiv \operatorname{prod}\left(X_{f} \mid f \in F\right)$.

Let $\tau \equiv\left[\tau_{0}, \ldots, \tau_{k}\right]$ be a second-order type and $k \geq 0$.

If $\mu \in M(\tau)$, then $\tau_{\mu}=\langle i, 1\rangle$ for some $i$. So $\tau_{\mu}(X)=A_{i}=\prod\left(A_{i f} \mid f \in F\right)=\prod\left(\tau_{\mu}\left(X_{f}\right) \mid f \in F\right)$. If $\nu \in N(\tau)$ then $\tau_{v}=\langle j, 0\rangle$ for some $j$. So $\tau_{v}(X)=K_{j}=\tau_{v}\left(X_{f}\right)$ for every $f \in F$. This means that the terminals of different parent types of the type $\tau$ over the formation $X$ have quite different constitutions. Therefore it is convenient to introduce the following notation. For elements $p \in \breve{\tau}(X)=\tau_{0}(X) \times \cdots \times \tau_{k}(X)$ and $f \in F$ define the element $p(f) \in \check{\tau}\left(X_{f}\right)=\tau_{0}\left(X_{f}\right) \times \cdots \times \tau_{k}\left(X_{f}\right)$ setting $p(f)(\mu) \equiv p(\mu)(f)$ for every $\mu \in M(\tau)$ and $p(f)(v) \equiv p(v)$ for every $v \in N(\tau))$.

For elements $P \subset \check{\tau}(X)$ and $f \in F$ define the element $P\langle f\rangle \subset \breve{\tau}\left(X_{f}\right)$ setting $P\langle f\rangle \equiv\left\{\xi \in \check{\tau}\left(X_{f}\right) \mid \exists p \in P(p(f)=\xi)\right\}$.

Let $\mathcal{D}$ be a subset of the power-set $\mathcal{P}(F)$. Define the superstructure $S$ of the signature $\Sigma_{2}^{g}$ over the formation $X$.

At first define the constant structures $s_{\omega}^{\tau} \in \tau(X)$ for $\tau \in \Theta$ and $\omega \in \Omega_{\tau}$.

If $\tau$ is a basic first-order type, then $\tau(X)=\prod\left(\tau\left(X_{f}\right) \mid f \in F\right)$. Therefore define $s_{\omega}^{\tau} \in \tau(X)$ setting $s_{\omega}^{\tau}\left(X_{f}\right) \equiv s_{\omega f}^{\tau}$ for every $f \in F$. If $\tau$ is an auxiliary first-order type, then $\tau(X)=\tau\left(X_{f}\right)$ and $s_{\omega f}^{\tau}$ does not depend on the index $f$. Therefore put $s_{\omega}^{\tau} \equiv s_{\omega f}^{\tau}$ for some (and then for every) $f \in F$.

If $\tau=\left[\tau_{0}, \ldots, \tau_{k}\right]$ is a second-order type, then put $s_{\omega f}^{\tau} \equiv\left\{p \in \check{\tau}(X) \mid \forall f \in F\left(p(f) \in s_{\omega}^{\tau}\right)\right\}$.

In result we obtain the collections $S_{c}^{\tau} \equiv\left(s_{\omega}^{\tau} \mid \omega \in \Omega_{\tau}\right)$ and the collection $S_{c} \equiv\left(S_{c}^{\tau} \mid \tau \in \Theta\right)$.

Now define the generalized equalities $\approx_{\tau} \subset \tau(X) \times \tau(X)$.

If $\tau$ is a basic first-order type, then for $p, q \in \tau(X)$ put $p \approx_{\tau} q$ iff $\exists G \in \mathcal{D} \exists g \in G\left(p(g) \approx_{\tau, g} q(g)\right.$ ). If $\tau$ is an auxiliary first-order type, then the equality $\approx_{\tau, f}$ does not depend on the index $f$. Therefore for $p, q \in \tau(X)$ put $p \approx_{\tau} q$ iff $p \approx_{\tau, f} q$ for some (and then for every) $f \in F$.

If $\tau=\left[\tau_{0}, \ldots, \tau_{k}\right]$ is a second-order type, then for $P, Q \subset \breve{\tau}(X)$ put $P \approx_{\tau} Q$ iff $\exists G \in \mathcal{D} \forall g \in G\left(P\langle g\rangle \approx_{\tau, g} Q\langle g\rangle\right)$.

In result we obtain the collection $S_{e} \equiv\left(\approx_{\tau} \mid \tau \in \Theta\right)$.

Now define the generalized belongings $<_{\tau} \subset \check{\tau}(X) \times \tau(X)$. Let $\tau \in \Theta_{b}$. By definition, $\tau=\left[\tau_{0}, \ldots, \tau_{k}\right]$ for some $\tau_{0}, \ldots, \tau_{k} \in \Theta$. For $p \in \check{\tau}(X)$ and $P \subset \breve{\tau}(X)$ put $p<_{\tau} P$ iff $\exists G \in \mathcal{D} \forall g \in G\left(p(g) \leftarrow_{\tau, g} P\langle g\rangle\right)$ (Note 1).

In result we obtain the collection $S_{b} \equiv\left(<_{\tau} \mid \tau \in \Theta_{b}\right)$.

Consider also the collection $S_{v} \equiv(\tau(X) \mid \tau \in \Theta)$ consisting of the $\tau$-terminals of the formation $X$.

The constructed collections compose the superstructure $S \equiv\left(S_{c}, S_{e}, S_{b}, S_{v}\right)$ over the formation $X$. Therefore we can consider the mathematical system $U \equiv(X, S)$ of the signature $\Sigma_{2}^{g}$. It will be called the infra-D-product of the collection of mathematical systems $\left(U_{f} \mid f \in F\right)$ of the generalized second-order signature $\Sigma_{2}^{g}$ and will be denoted by infra-D- $\operatorname{prod}\left(U_{f} \mid f \in F\right)$.

Further we assume that $\mathcal{D}$ is a filter.

Now let $\left(\left(U_{f}, \gamma_{f}\right) \mid f \in F\right)$ be a pairwise $H$-concordant collection of evaluated mathematical systems of the second-order signature $\Sigma_{2}^{g}$ with true generalized equalities and belongings.

Define the evaluation $\gamma$ on the system $U \equiv \operatorname{infra-} \mathcal{D}-\operatorname{prod}\left(U_{f} \mid f \in F\right)$ in the following way.

Let $x$ be a variable of a type $\tau$. If $\tau$ is a first-order basic type, then define $\gamma(x) \in \tau(X)$ setting $\gamma(x)(f) \equiv \gamma_{f}(x)$ for every $f \in F$. If $\tau$ is an auxiliary first-order type, then put $\gamma(x) \equiv \gamma_{f}(x)$ for some (and then for every) $f \in F$.

If $\tau=\left[\tau_{0}, \ldots, \tau_{k}\right]$ is a second-order type, then put $\gamma(x) \equiv\left\{p \in \check{\tau}(X) \mid \forall f \in F\left(p(f) \in \gamma_{f}(x)\right)\right\}$.

The evaluation $\gamma$ will be called the crossing of the collection of evaluations $\left(\gamma_{f} \mid f \in F\right)$ and will be denoted by $\bowtie\left(\gamma_{f} \mid f \in F\right)$.

Lemma 2 Let $\left(U_{f} \mid f \in F\right)$ be a pairwise H-concordant collection of mathematical systems of the second-order signature $\Sigma_{2}^{g}$ and let every evaluated mathematical system $\left(U_{f}, \gamma_{f}\right)$ be a model for equality axioms $E 1-E 4$. Then the pair (infra-DD-prod $\left(U_{f} \mid f \in F\right), \bowtie\left(\gamma_{f} \mid f \in F\right)$ ) is also a model for axioms $E 1-E 4$.

Proof. Let $t_{0}, t_{0}^{\prime} \in \tau_{0}(X), \ldots, t_{k}, t_{k}^{\prime} \in \tau_{k}(X), P, P^{\prime} \subset \breve{\tau}(X)=\tau_{0}(X) \times \ldots \times \tau_{k}(X), p \equiv\left(t_{0}, \ldots, t_{k}\right), p^{\prime} \equiv\left(t_{0}^{\prime}, \ldots, t_{k}^{\prime}\right)$, $p \approx_{\breve{\tau}} p^{\prime}$ and $P \approx_{\tau} P^{\prime}$.

Assume that $p<_{\tau} P$. By the definition of the belonging $\exists G_{1} \in \mathcal{D} \forall g \in G_{1}\left(p(q)<_{\tau, g} P\langle q\rangle\right)$. By the definition of the 


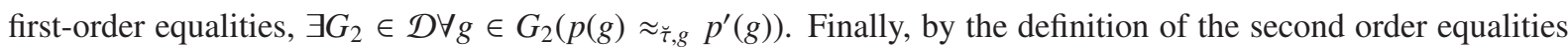
$\exists G_{3} \in \mathcal{D} \forall g \in G_{3}\left(P\langle g\rangle \approx_{\tau, g} P^{\prime}\langle g\rangle\right)$. Since every system $\left(U_{g}, \gamma_{g}\right)$ satisfies $E 4$, we see that $p^{\prime}(g)<_{\tau, g} P^{\prime}\langle g\rangle$ for every $g \in G \equiv G_{1} \cap G_{2} \cap G_{3}$. So $p^{\prime}<_{\tau} P^{\prime}$. Hence, $p<_{\tau} P \Rightarrow p^{\prime}<_{\tau} P^{\prime}$. The inverse implication is checked quite similarly. This proves axiom $E 4$. The validity of axioms E1, E2, E3 is obvious. $\triangle$

Further for a formula $\varphi \in L(\Sigma)$ the set $\left\{f \in F \mid U_{f} \vDash \varphi\left[\gamma_{f}\right]\right\}$ will be denoted by $G_{\varphi}$.

Lemma 3 Let $\tau=\left[\tau_{0}, \ldots, \tau_{k}\right]$ be a second-order type. Let $s_{\omega}^{\tau}$ be the constants constructed above for the support $X \equiv \operatorname{prod}\left(X_{f} \mid f \in F\right)$. Then $s_{\omega}^{\tau}\langle f\rangle=s_{\omega f}^{\tau}$ for every $f \in F$.

Proof. Let $\xi \in s_{\omega}^{\tau}\langle f\rangle$, i.e., $\xi=p(f)$ for some $p \in s_{\omega}^{\tau}$. By definition, $\xi=p(f) \in s_{\omega f}^{\tau}$. Consequently, $s_{\omega}^{\tau}(f) \subset s_{\omega f}^{\tau}$.

Conversely, let $\xi_{f} \in s_{\omega f}^{\tau}$. Since the collection of systems $\left(U_{f} \mid f \in F\right)$ is $H$-concordant, using the axiom of choice we can find a collection $\left(\xi_{g} \mid g \in F \backslash\{f\}\right)$ such that $\xi_{g} \in s_{\omega g}^{\tau}$ and $\xi_{g}(v)=\xi_{f}(v)$ for every $v \in N(\tau)$. Define the element $p \in \breve{\tau}(X)$ setting $p(\mu)(g) \equiv \xi_{g}(\mu)$ for every $g \in F$ and every $\mu \in M(\tau)$ and $p(v) \equiv \xi_{f}(v)$ for every $v \in N(\tau)$. Then $p(g)=\xi_{g} \in s_{\omega g}^{\tau}$ for every $g \in F$ implies $p \in s_{\omega}^{\tau}$. Since $\xi_{f}=p(f)$, we have $\xi_{f} \in s_{\omega}^{\tau}\langle f\rangle$. Hence, $s_{\omega f}^{\tau} \subset s_{\omega}^{\tau}\langle f\rangle$. $\triangle$

Lemma 4 Let $\tau=\left[\tau_{0}, \ldots, \tau_{k}\right]$ be a second-order type. Let $x$ be a variable of the type $\tau$ and $\gamma(x)$ be the evaluation constructed above for the system $U \equiv(X, S)$. Then $\gamma(x)\langle f\rangle=\gamma_{f}(x)$ for every $f \in F$.

The proof is completely similar to the proof of the previous lemma.

\section{Infrafilteration of Formulas of the Second-Order Language $L\left(\Sigma_{2}^{g}\right)$ of the Generalized Second-Order Sig- nature $\Sigma_{2}^{g}$}

Consider a non-empty set $F$ and a filter $\mathcal{D}$ on $F$.

By analogy with the first order language (Ershov \& Palyutin, 1984, §17; Mal'cev, 1970, 8.2) a formula $\varphi$ of the language $L\left(\Sigma_{2}^{g}\right)$ of the second-order signature $\Sigma_{2}^{g}$ with generalized equalities and belongings will be called infrafiltrated with respect to the filter $\mathcal{D}$ if for every pairwise $H$-concordant collection $\left(\left(U_{f}, \gamma_{f}\right) \mid f \in F\right)$ of evaluated mathematical systems of the second-order signature $\Sigma_{2}^{g}$ with true generalized equalities and belongings the property infra-D $\mathcal{D}-\operatorname{prod}\left(U_{f} \mid f \in F\right) \vDash \varphi\left[\bowtie\left(\gamma_{f} \mid f \in F\right)\right]$ is equivalent to the property $\left\{g \in F \mid U_{g} \vDash \varphi\left[\gamma_{g}\right]\right\} \in \mathcal{D}$.

Lemma 5 Every atomic formula is infrafiltered with respect to any filter $\mathcal{D}$ on the set $F$.

Proof. At first consider an atomic formula $\varphi$ of the form $q^{\tau} \delta_{\tau} r^{\tau}$. Assume that $q^{\tau}=x^{\tau}$ and $r^{\tau}=\sigma_{\omega}^{\tau}$. Then $U \models \varphi[\gamma]$ is equivalent to $\gamma(x) \approx_{\tau} s_{\omega}^{\tau}$, and analogously for the pair $\left(U_{f}, \gamma_{f}\right)$.

Let $\tau$ be a first-order type. Let $G_{\varphi} \in \mathcal{D}$, i.e., $\gamma_{g}(x) \approx_{\tau, g} s_{\omega g}^{\tau}$ for every $g \in G_{\varphi} \in \mathcal{D}$. If $\tau$ is a basic type, then $\gamma_{g}(x)=\gamma(x)(g)$ and $s_{\omega g}^{\tau}$ implies $\gamma(x)(g) \approx_{\tau, g} s_{\omega}^{\tau}(g)$ for every $g \in G_{\varphi} \in \mathcal{D}$. So $\gamma(x) \approx_{\tau} s_{\omega}^{\tau}$. If $\tau$ is an auxiliary type, then $\gamma_{g}(x)=\gamma(x)$ and $s_{\omega g}^{\tau}=s_{\omega}^{\tau}$. Besides, $\approx_{\tau, g}$ coincides with $\approx_{\tau}$. Hence $\gamma(x) \approx_{\tau} s_{\omega}^{\tau}$. In both cases we have obtained the property $U \vDash \varphi[\gamma]$.

Conversely, let $U \models \varphi[\gamma]$, i.e., $\gamma(x) \approx_{\tau} s_{\omega}^{\tau}$. If $\tau$ is a basic type, then there exists $G \in \mathcal{D}$ such that $\gamma(x)(g) \approx_{\tau, g} s_{\omega}^{\tau}(g)$ for every $g \in G$. But it means that $\gamma_{g}(x) \approx_{\tau, g} s_{\omega g}^{\tau}$, i.e., $U_{g} \vDash \varphi\left[\gamma_{g}\right]$ for every $g \in G \in \mathcal{D}$. Since $G \subset G_{\varphi}$, we have $G_{\varphi} \in \mathcal{D}$. If $\tau$ is an auxiliary type then $\gamma_{f}(x) \approx_{\tau, f} s_{\omega f}^{\tau}$ for every $f \in F$. Consequently, $G_{\varphi} \in \mathcal{G}$ again.

Now let $\tau \equiv\left[\tau_{0}, \ldots, \tau_{k}\right]$ be a second-order type. Let $G_{\varphi} \in \mathcal{D}$, i.e., $\gamma_{g}(x) \approx_{\tau, g} s_{\omega g}^{\tau}$ for every $g \in G_{\varphi} \in \mathcal{D}$. According to Lemmas 3 and 4 the equalities $s_{\omega g}^{\tau}=s_{\omega}^{\tau}\langle g\rangle$ and $\gamma_{g}(x)=\gamma(x)\langle g\rangle$ are correct. Therefore $\gamma(x)\langle g\rangle \approx_{\tau, g} s_{\omega}^{\tau}\langle g\rangle$ for every $G_{\varphi} \in \mathcal{D}$. Consequently, $\gamma(x) \approx_{\tau} s_{\omega}^{\tau}$, i.e., $U \models \varphi[\gamma]$.

Conversely, let $U \vDash \varphi[\gamma]$, i.e., $\gamma(x) \approx_{\tau} s_{\omega}^{\tau}$. By the definition of the second-order equality $\gamma(x)\langle g\rangle \approx_{\tau, g} s_{\omega}^{\tau}\langle g\rangle$ for some $G \in \mathcal{D}$ and every $g \in G$. Using Lemmas 3 and 4 we obtain $\gamma_{g}(x) \approx_{\tau, g} s_{\omega g}^{\tau}$, i.e., $U_{g} \vDash \varphi\left[\gamma_{g}\right]$ for every $g \in G$. Since $G \subset G_{\varphi}$, we infer that $G_{\varphi} \in \mathcal{D}$.

For terms $q^{\tau}$ and $r^{\tau}$ of other forms the reasons are quite similar.

Now consider an atomic formula $\varphi$ of the form $\left(q_{0}^{\tau_{0}}, \ldots, q_{k}^{\tau_{k}}\right) \varepsilon_{\tau} r^{\tau}$ for $\tau \equiv\left[\tau_{0}, \ldots, \tau_{k}\right] \in \Theta_{b}$. Assume that $q_{\lambda}^{\tau_{\lambda}}=x_{\lambda}^{\tau \lambda}$ and $r^{\tau}=u^{\tau}$ for some variables $x_{\lambda}$ and $u$. Then $U \models \varphi[\gamma]$ is equivalent to $\left(\gamma\left(x_{0}\right), \ldots, \gamma\left(x_{k}\right)\right)<_{\tau} \gamma(u)$ and analogously for the pair $\left(U_{f}, \gamma_{f}\right)$.

Let $G_{\varphi} \in \mathcal{D}$, i.e., $\left(\gamma_{g}\left(x_{0}\right), \ldots, \gamma_{g}\left(x_{k}\right)\right)<_{\tau, g} \gamma_{g}(u)$ for every $g \in G \varphi \in \mathcal{D}$. Consider the elements $\xi_{f} \equiv\left(\gamma_{f}\left(x_{0}\right), \ldots, \gamma_{f}\left(x_{k}\right)\right)$ and $p \equiv\left(\gamma\left(x_{0}\right), \ldots, \gamma\left(x_{k}\right)\right) \in \breve{\tau}(X)$. Let $f \in F$. Then $p(f)(\mu) \equiv p(\mu)(f)=\gamma\left(x_{\mu}\right)(f)=\gamma_{f}\left(x_{\mu}\right)=\xi_{f}(\mu)$ for every $\mu \in M(\tau)$ and $p(f)(v) \equiv p(v)=\gamma\left(x_{v}\right)=\gamma_{f}\left(x_{v}\right)=\xi_{f}(v)$ for every $v \in N(\tau)$. Consequently, $p(f)=\xi_{f}$. By Lemma $3 \gamma_{f}(u)=\gamma(u)\langle f\rangle$. In result we obtain $p(g) \epsilon_{\tau, g} \gamma(u)\langle g\rangle$ for every $g \in G_{\varphi} \in \mathcal{D}$. By definition, it means that $p<_{\tau} \gamma(u)$, 
i.e., $U \vDash \varphi[\gamma]$.

Conversely, let $U \vDash \varphi[\gamma]$, i.e., $\left(\gamma\left(x_{0}\right), \ldots, \gamma\left(x_{k}\right)\right)<_{\tau} \gamma(u)$. By the definition of the second-order belonging for $p \equiv$ $\left(\gamma\left(x_{0}\right), \ldots, \gamma\left(x_{k}\right)\right)$ there exists $G \in \mathcal{D}$ such that $p(g) \leftarrow_{\tau, g} \gamma(u)\langle g\rangle$ for every $g \in G$. By Lemma $4 \gamma(u)\langle g\rangle=\gamma_{g}(x)$. By the previous paragraph $\xi_{g}=p(g)$. Consequently, $\xi_{g} \leftarrow_{\tau, g} \gamma_{g}(u)$, i.e., $U_{g} \vDash \varphi\left[\gamma_{g}\right]$ for every $g \in G$. Since $G \subset G_{\varphi}$, we infer that $G_{\varphi} \in \mathcal{D}$.

For terms $q_{\lambda}^{\tau_{\lambda}}$ and $r^{\tau}$ of other forms the reasons are quite similar.

A proof of the property of infrafiltration for the quantified formula $\exists x^{\tau} \varphi$ for the polygrade language $L\left(\Sigma_{2}^{g}\right)$ of the generalized second-order signature $\Sigma_{2}^{g}$ is more delicate than for the first-order language. Therefore we begin it with a subsidiary proposition.

Let $\left(\left(U_{f}, \gamma_{f}\right) \mid f \in F\right)$ be a pairwise $H$-concordant collection of evaluated mathematical systems of the secondorder signature $\Sigma_{2}^{g}$ with true generalized equalities and belongings. Let $\beta$ be an evaluation on the system $U \equiv$ infra- $\mathcal{D}-\operatorname{prod}\left(U_{f} \mid f \in F\right), H$-concordant with the evaluation $\gamma \equiv \bowtie\left(\gamma_{f} \mid f \in F\right)$.

With respect to the evaluation $\beta$ for every $f \in F$ define the evaluation $\delta_{f}$ on the system $U_{f}$ in the following way. Let $x$ be a variable of a type $\tau$. If $\tau$ is a basic first-order type, then put $\delta_{f}(x) \equiv \beta(x)(f)$. If $\tau$ is an auxiliary first-order type, then put $\delta_{f}(x) \equiv \beta(x)$. If $\tau$ is a second-order type, then put $\delta_{f}(x) \equiv \beta(x)\langle f\rangle$.

Proposition 41$)$ The collection $\left(\left(U_{f}, \delta_{f}\right) \mid f \in F\right)$ of the evaluated mathematical systems $\left(U_{f}, \delta_{f}\right)$ of the secondorder signature $\Sigma_{2}^{g}$ with true generalized equalities and belongings is pairwise $H$-concordant;

2) the evaluated systems $\left(U_{f}, \gamma_{f}\right)$ and $\left(U_{f}, \delta_{f}\right)$ are $H$-concordant;

3) for the evaluation $\delta \equiv \bowtie\left(\delta_{f} \mid f \in F\right)$ the equalities $\delta\left(x^{\tau}\right) \approx_{\tau} \beta\left(x^{\tau}\right)$ hold for any variable $x^{\tau}$;

4) the evaluations $\delta$ and $\beta$ are $H$-concordant.

Proof. 1) Let $x$ be a variable of a type $\tau$. If $\tau$ is an auxiliary first-order type, then $\delta_{f}(x)=\beta(x)=\delta_{g}(x)$ for every $f, g \in F$.

Let $\tau \equiv\left[\tau_{0}, \ldots, \tau_{k}\right]$ be a second-order type. Fix some $f, g \in F$. Consider an arbitrary element $\xi \in \delta_{f}(x)=\beta(x)\langle f\rangle$. By definition, $\xi=p(f)$ for some $p \in \beta(x) \subset \breve{\tau}(X)=\tau_{0}(X) \times \ldots \times \tau_{k}(X)$. Consider the element $\eta \equiv p(g) \in \beta(x)\langle g\rangle=$ $\delta_{g}(x)$. Then $\eta(v)=p(g)(v)=p(v)$ and $\xi(v)=p(f)(v)=p(v)$ implies $\eta(v)=\xi(v)$ for every $v \in N(\tau)$. The inverse finding the element $\xi$ corresponding to the given element $\eta$ is realized in the similar manner.

2) If $\tau$ is an auxiliary first-order type, then $\delta_{f}\left(x^{\tau}\right) \equiv \beta\left(x^{\tau}\right)$ and $\gamma\left(x^{\tau}\right) \equiv \gamma_{f}\left(x^{\tau}\right)$. By condition, $\beta\left(x^{\tau}\right)=\gamma\left(x^{\tau}\right)$. Consequently, $\delta_{f}\left(x^{\tau}\right)=\gamma_{f}\left(x^{\tau}\right)$.

Let $\tau \equiv\left[\tau_{0}, \ldots, \tau_{k}\right]$ be a second-order type. Consider an arbitrary element $\xi \in \gamma_{f}(x)$. In virtue of Lemma 4 we have $\gamma_{f}(x)=\gamma(x)\langle f\rangle$. Since $\xi \in \gamma(x)\langle f\rangle$, by definition there exists $p \in \gamma(x)$ such that $\xi=p(f)$. By condition, for $p \in \gamma(x)$ there is $q \in \beta(x)$ such that $q(v)=p(v)$ for any $v \in N(\tau)$. Consider the element $\eta \equiv q(f) \in \beta(x)\langle f\rangle=\delta_{f}(x)$. Then $\eta(v)=q(f)(v)=q(v)=p(v)=p(f)(v)=\xi(v)$. The inverse condition is checked in the same way.

3) Let $x$ be a variable of a type $\tau$. If $\tau$ is a basic first-order type, then by the definition of the evaluations $\delta$ and $\delta_{f}$ we obtain $\delta(x)(f) \equiv \delta_{f}(x)=\beta(x)(f)$ for any $f \in F$, i.e., $\delta(x)=\beta(x)$. If $\tau$ is an auxiliary first-order type, then $\delta(x)=\delta_{f}(x)=\beta(x)$ for some $f \in F$.

Let $\tau$ be a second-order type. Then $\delta(x)=\prod\left(\delta_{f}(x) \mid f \in F\right)$. In virtue of Lemma $4 \delta(x)\langle f\rangle=\delta_{f}(x)=\beta(x)\langle f\rangle$ for any $f \in F$. By the definition of the second-order equality, we conclude that $\delta(x) \approx_{\tau}(\beta)(x)$.

4) Let $\tau$ be an auxiliary first-order type. Then $\delta\left(x^{\tau}\right)=\delta_{f}\left(x^{\tau}\right)$ for some $f \in F$. By definition, $\delta_{f}\left(x^{\tau}\right)=\beta\left(x^{\tau}\right)$. Consequently, $\delta\left(x^{\tau}\right)=\beta\left(x^{\tau}\right)$.

Let $\tau=\left[\tau_{0}, \ldots, \tau_{k}\right]$ be a second-order type. Let $p \in \beta(x)$. By the definition of the cut, $p(f) \in \beta(x)\langle f\rangle=\delta_{f}(x)$ for every $f \in F$. By the definition of the crossing, $p \in \delta(x)$. So for $p \in \beta(x)$ there exists $q=p \in \delta(x)$ such that $q(v)=p(v)$ for every $v \in N(\tau)$.

Conversely, let $q \in \delta\left(x^{\tau}\right)$. By the definition of the crossing, $q(f) \in \delta_{f}(x)=\beta(x)\langle f\rangle$ for every $f \in F$. Fix some element $f_{0} \in F$. By the definition of the cut, there is $p \in \beta(x)$ such that $p\left(f_{0}\right)=q\left(f_{0}\right)$. If $v \in N(\tau)$, then $p\left(f_{0}\right)(v)=q\left(f_{0}\right)(v)$. However $p\left(f_{0}\right)(v)=p(v)$ and $q\left(f_{0}\right)(v)=q(v)$. Therefore $p(v)=q(v)$ for every $v \in N(\tau)$.

Proposition 5 Let a formula $\psi$ be infrafiltrated with respect to the filter $\mathcal{D}$. Then the formula $\exists x^{\tau} \psi$ is infrafiltrated by $\mathcal{D}$ too. 
Proof. Denote the formula $\exists x^{\tau} \psi$ by $\varphi$. Let $G_{\varphi} \in \mathcal{D}$, i.e., $U_{g} \vDash \varphi\left[\gamma_{g}\right]$ for every $g \in G_{\varphi} \in \mathcal{D}$. Further we shall write simply $G$ instead of $G_{\varphi}$.

The presented satisfaction property means that $U_{g} \vDash \psi\left[\gamma_{g}^{\prime}\right]$ for some evaluation $\gamma_{g}^{\prime}, H$-concordant with the evaluation $\gamma_{g}$ and such that $\gamma_{g}^{\prime}(y)=\gamma_{g}(y)$ for every $y^{\sigma} \neq x^{\tau}$. For every $f \in F$ define the evaluation $\delta_{f}$ setting $\delta_{f} \equiv \gamma_{f}$ if $f \in F \backslash G$ and $\delta_{f} \equiv \gamma_{f}^{\prime}$ if $f \in G$. Check that the evaluated systems $\left(U_{f}, \delta_{f}\right)$ and $\left(U_{g}, \delta_{g}\right)$ are $H$-concordant for every $f, g \in F$. If $f, g \in F \backslash G$, then $\delta_{f}=\gamma_{f}$ and $\delta_{g}=\gamma_{g}$. Since the evaluations $\gamma_{f}$ and $\gamma_{g}$ are $H$-concordant, our assertion is true. Let $f, g \in G$. Then $\delta_{f}=\gamma_{f}^{\prime}$ and $\delta_{g}=\gamma_{g}^{\prime}$. Let $x$ be a variable of a type $\tau$.

For an auxiliary first-order type $\tau$ we have $\gamma_{f}^{\prime}(x)=\gamma_{f}(x)$ and $\gamma_{g}^{\prime}(x)=\gamma_{g}(x)$. Since the evaluations $\gamma_{f}$ and $\gamma_{g}$ are $H$-concordant, we infer that $\gamma_{f}(x)=\gamma_{g}(x)$. Consequently $\delta_{f}(x)=\gamma_{f}^{\prime}(x)=\gamma_{g}^{\prime}(x)=\delta_{g}(x)$.

Let $\tau$ be a second-order type. Let $p \in \delta_{f}(x)=\gamma_{f}^{\prime}(x)$. Then there exists $q \in \gamma_{f}(x)$ such that $q(v)=p(v)$ for every $v \in N(\tau)$. Since the evaluations $\gamma_{f}$ and $\gamma_{g}$ are $H$-concordant, there is $r \in \gamma_{g}(x)$ such that $r(v)=q(v)$. Since the evaluations $\gamma_{g}$ and $\gamma_{g}^{\prime}$ are $H$-concordant as well, there exists $s \in \gamma_{g}^{\prime}(x)=\delta_{g}(x)$ such that $s(v)=r(v)=q(v)=p(v)$ for any $v \in N(\tau)$. The inverse condition is checked in the same way.

In the cases when $f \in F \backslash G$ and $g \in G$ or conversely the arguments are similar.

So the collection $\left(\left(U_{f}, \delta_{f}\right) \mid f \in F\right)$ of evaluated mathematical systems of the signature $\Sigma_{2}^{g}$ with true generalized equalities and belongings is pairwise $H$-concordant. Consider the evaluation $\delta \equiv \bowtie\left(\delta_{f} \mid f \in F\right)$.

Check that $\delta(y)=\gamma(y)$ for every $y^{\sigma} \neq x^{\tau}$. Let $\sigma$ be a basic first-order type. If $g \in G$, then $\delta(y)(g)=\delta_{g}(y)=\gamma_{g}^{\prime}(y)=$ $\gamma_{g}(y)=\gamma(y)(g)$. If $f \in F \backslash G$, then $\delta(y)(f)=\delta_{f}(y)=\gamma_{f}(y)=\gamma(y)(f)$. Consequently, $\delta(y)=\gamma(y)$.

Let $\sigma$ be an auxiliary first-order type. Then $\delta(y)=\delta_{f}(y)=\gamma_{f}(y)=\gamma(y)$ for some $f \in F \backslash G$.

Let $\sigma$ be a second-order type. Then $\delta(y)=\prod\left(\delta_{f}(y) \mid f \in F\right)$. If $f \in G$, then $\delta_{f}(y)=\gamma_{f}^{\prime}(y)=\gamma_{f}(y)$. If $f \in F \backslash G$, then $\delta_{f}(y)=\gamma_{f}(y)$. So $\delta(y)=\prod\left(\gamma_{f}(y) \mid f \in F\right)=\gamma(y)$.

Thus for every $y \neq x$ we have $\delta(y)=\gamma(y)$.

Check that the evaluations $\gamma$ and $\delta$ are $H$-concordant. Let $y^{\sigma} \neq x^{\tau}$.

If $\sigma$ in an auxiliary first-order type, then $\delta(y)=\gamma(y)$.

Let $\sigma$ be a second-order type. It was proved above that $\delta(y)=\gamma(y)$. Consequently for every $p \in \delta(y)$ there is $q \equiv p \in \gamma(y)$ such that $q(v)=p(v)$ for any $v \in N(\sigma)$.

If $\tau$ is an auxiliary first-order type, then $\delta(x)=\delta_{f}(x)=\gamma_{f}(x)=\gamma(x)$ for some $f \in F \backslash G$.

Let $\tau$ be a second-order type. Let $p \in \gamma\left(x^{\tau}\right)=\prod\left(\gamma_{f}(x) \mid f \in F\right)$. If $f \in F \backslash G$, then $\delta_{f}=\gamma_{f}$. If $g \in G$, then $\delta_{g}=\gamma_{g}^{\prime}$ and the evaluations $\gamma_{g}^{\prime}$ and $\gamma_{g}$ are $H$-concordant.

Consider the nonempty set $A \equiv \bigcup\left(\gamma_{g}^{\prime}(x) \mid g \in G\right)$. Define the mapping $\alpha: G \rightarrow \mathcal{P}(A) \backslash\{\varnothing\}$ setting $\alpha(g) \equiv\{\eta \in$ $\left.\gamma_{g}^{\prime}(x) \subset A \mid \forall v \in N(\tau)(\eta(v)=p(g)(v))\right\}$. According to the point 3 of the definition of $H$-concordant systems the set $\alpha(g)$ is non-empty.

By the axiom of choice there exists a function $c h: \mathcal{P}(A) \backslash\{\varnothing\} \rightarrow A$ such that $\operatorname{ch} P \in P$. Consider the function $\beta \equiv \operatorname{ch} \circ \alpha: G \rightarrow A$ and the corresponding collection $\beta=\left(\eta_{g} \in A \mid g \in G\right)$. Since $\eta_{g}=\beta(g)=\operatorname{ch}(\alpha(g)) \in \alpha(g)$ then, we have $\eta_{g}(v)=p(g)(v)=p(v)$ for every $v \in N(\tau)$.

Define the element $q \in \check{\tau}(X)$ setting $q(\mu)(f) \equiv p(\mu)(f)$ for every $f \in F \backslash G, q(\mu)(g) \equiv \eta_{g}(\mu)$ for every $g \in G$ and every $\mu \in M(\tau)$, and $q(v) \equiv \eta_{g}(v)=p(v)$ for every $v \in N(\tau)$ and every $g \in G$.

Then $q(f)(\mu)=q(\mu)(f)=p(\mu)(f)=p(f)(\mu)$ for every $\mu \in M(\tau)$ and $q(f)(v)=q(v)=p(v)=p(f)(v)$ for every $v \in N(\tau)$ implies $q(f)=p(f) \in \gamma_{f}(x)=\delta_{f}(x)$ for every $f \in F \backslash G$. If $g \in G$, then $q(g)(\mu)=q(\mu)(g)=\eta_{g}(\mu)$ for every $\mu \in M(\tau)$ and $q(g)(v)=q(v)=\eta_{g}(v)$ for every $v \in N(\tau)$ implies $q(g)=\eta_{g} \in \gamma_{g}^{\prime}(x)=\delta_{g}(x)$. Consequently, $q \in \prod\left(\delta_{f}(x) \mid f \in F\right)=\delta\left(x^{\tau}\right)$. Besides, $q(v)=p(v)$ for every $v \in N(\tau)$. The inverse finding the element $p$ corresponding to the given element $q$ is realized in the similar manner.

So the evaluations $\gamma$ and $\delta$ are really $H$-concordant.

By condition and construction, $U_{g} \vDash \psi\left[\delta_{g}\right]$ for every $g \in G \in \mathcal{D}$. Since the formula $\psi$ is infrafiltered, the obtained property implies the property $U \models \psi[\delta]$. Since the evaluation $\delta$ is $H$-concordant with the evaluation $\gamma$ and $\delta\left(y^{\sigma}\right)=\gamma\left(y^{\sigma}\right)$ for every $y^{\sigma} \neq x^{\tau}$, we obtain the property $U \vDash \varphi[\gamma]$. 
Conversely, let $U \models \varphi[\gamma]$. It is equivalent to $U \models \psi[\beta]$ for some evaluation $\beta, H$-concordant with the evaluation $\gamma$ and such that $\beta(y)=\gamma(y)$ for every $y^{\sigma} \neq x^{\tau}$.

Consider the evaluation $\delta \equiv \bowtie\left(\delta_{f} \mid f \in F\right)$ from Proposition 4, corresponding to the evaluation $\beta$. According to Proposition 4 the evaluations $\delta$ and $\beta$ are $H$-concordant and $\delta\left(z^{\rho}\right) \approx_{\rho} \beta\left(z^{\rho}\right)$ for every variable $z^{\rho}$. It follows from Proposition 2 that the property $U \models \psi[\beta]$ is equivalent to the property $U \models \psi[\delta]$. Since the formula $\psi$ is infrafiltrated, the property $U \models \psi[\delta]$ is equivalent to the property $G \equiv\left\{g \in F \mid U_{g} \models \psi\left[\delta_{g}\right]\right\} \in \mathcal{D}$.

By Proposition 4 the evaluations $\delta_{g}$ and $\gamma_{g}$ are $H$-concordant. Let $y^{\sigma} \neq x^{\tau}$. If $\sigma$ is a basic first-order type, then $\delta_{g}(y)=\beta(y)(g)=\gamma(y)(g)=\gamma_{g}(y)$. If $\sigma$ is an auxiliary first-order type, then $\delta_{g}(y)=\beta(y)=\gamma(y)=\gamma_{g}(y)$. Finally, if $\sigma$ is a second-order type, then $\delta_{g}(y)=\beta(y)\langle g\rangle=\gamma(y)\langle g\rangle$. Since by Lemma $4 \gamma(y)\langle g\rangle=\gamma_{g}(y)$, we have $\delta_{g}(y)=\gamma_{g}(y)$. Consequently in all the cases $\delta_{g}(y)=\gamma_{g}(y)$ for every $y^{\sigma} \neq x^{\tau}$. Therefore the property $U_{g} \vDash \psi\left[\delta_{g}\right]$ is equivalent to the property $U_{g} \vDash \varphi\left[\gamma_{g}\right]$. So $\left\{g \in F \mid U_{g} \vDash \varphi\left[\gamma_{g}\right]\right\}=G \in \mathcal{D}$. This implies $G_{\varphi} \in \mathcal{D}$.

The following two lemmas are the same as ones for the first-order language.

Lemma 6 Let formulas $\psi$ and $\xi$ be infrafiltered with respect to the filter $\mathcal{D}$. Then the formula $\psi \wedge \xi$ is infrafiltered by $\mathcal{D}$ too.

Proof. Denote the formula $\psi \wedge \xi$ by $\varphi$. Let $G_{\varphi} \in \mathcal{D}$, i.e., $U_{g} \vDash \varphi\left[\gamma_{g}\right]$ for all $g \in G_{\varphi} \in \mathcal{D}$. This property is equivalent to conjunction of the properties $U_{g} \vDash \psi\left[\gamma_{g}\right]$ and $U_{g} \vDash \xi\left[\gamma_{g}\right]$. Since these formulas are infrafiltered, it is equivalent to conjunction of the properties $U \models \psi[\gamma]$ and $U \models \xi[\gamma]$, but it is equivalent to the property $U \models \varphi[\gamma]$.

Conversely, let $U \vDash \varphi[\gamma]$. It is equivalent to the conjunction of the properties $U \vDash \psi[\gamma]$ and $U \vDash \xi[\gamma]$. Then $G_{\psi} \in \mathcal{D}$ and $G_{\xi} \in \mathcal{D}$. Consider $G \equiv G_{\psi} \cap G_{\xi}$. Then $U_{g} \vDash \psi\left[\gamma_{g}\right]$ and $U_{g} \vDash \xi\left[\gamma_{g}\right]$ implies $U_{g} \vDash \varphi\left[\gamma_{g}\right]$ for every $g \in G \in \mathcal{D}$. Hence, $G_{\varphi} \in \mathcal{D}$.

Lemma 7 Let a formula $\psi$ be infrafiltered with respect to the ultrafilter $\mathcal{D}$. Then the formula $\neg \psi$ is infrafiltered by $\mathcal{D}$ too.

Proof. Denote the formula $\neg \psi$ by $\varphi$. By assumption, the properties $G_{\psi} \in \mathcal{D}$ and $U \models \psi[\gamma]$ are equivalent.

By definition, $F \backslash G_{\varphi}=\left\{g \in F \mid\right.$ the property $U_{g} \vDash \varphi\left[\gamma_{g}\right]$ does not hold $\}$. But $U_{g} \vDash \varphi\left[\gamma_{g}\right]$ is equivalent to the assertion that the property $U_{g} \vDash \psi\left[\gamma_{g}\right]$ does not hold. Consequently the property $U_{g} \vDash \psi\left[\gamma_{g}\right]$ is equivalent to the assertion that the property $U_{g} \vDash \varphi\left[\gamma_{g}\right]$ does not hold. It implies $F \backslash G_{\varphi}=G_{\psi}$.

Let $G_{\varphi} \in \mathcal{D}$. Since $\mathcal{D}$ is an ultrafilter, we have $G_{\psi}=F \backslash G_{\varphi} \notin \mathcal{D}$. So the property $U \vDash \psi[\gamma]$ does not hold. By the definition of the satisfiability, it means that $U \models \varphi[\gamma]$.

Conversely, let $U \models \varphi[\gamma]$. Then the property $U \models \psi[\gamma]$ does not hold. Therefore $G_{\psi} \notin \mathcal{D}$. Since $\mathcal{D}$ is an ultrafilter, we have $G_{\varphi}=F \backslash G_{\psi} \in \mathcal{D}$.

Theorem 1 Every formula $\varphi$ of the language $L\left(\Sigma_{2}^{g}\right)$ of the second-order signature $\Sigma_{2}^{g}$ with generalized equalities and belongings is infrafiltered with respect to any ultrafilter $\mathcal{D}$ on the set $F$.

Proof. The set of all formulas $\varphi$ of the language $L\left(\Sigma_{2}^{g}\right)$, constructed by induction from atomic formulas by means of the connectives $\neg$ and $\wedge$ and the quantifier $\exists$, will be denoted by $\Psi$. The subset of the set $\Psi$, consisting of all formulas containing at most $n$ logical symbols $\neg$, $\wedge$, and $\exists$, will be denoted by $\Psi_{n}$. Obviously, $\Psi=\bigcup\left(\Psi_{n} \mid n \in \omega_{0}\right)$.

Using the complete induction principle (see Mendelson, 1997, 3.1, Proposition 3.9) we shall prove the following assertion $A(n)$ : every formula $\varphi \in \Psi_{n}$ is infrafiltered.

If $n=0$, then $\varphi$ is an atomic formula. By Lemma 5 it is infrafiltered. Consequently, $A(0)$ holds.

Assume that for every $m<n$ the assertion $A(m)$ holds. Let $\varphi \in \Psi_{n}$. If $\varphi=\neg \psi$, then $\psi \in \Psi_{n-1}$. Therefore, $\psi$ is infrafiltered. By Lemma 7 the formula $\varphi$ is infrafiltered too. If $\varphi=\psi \wedge \xi$, then $\psi, \xi \in \Psi_{n-1}$. Therefore, by the inductive assumption, the formulas $\psi$ and $\xi$ are infrafiltered. By Lemma 6 the formula $\varphi$ is infrafiltered too. Finally, if $\varphi=\exists x^{\tau} \psi$, then $\psi \in \Psi_{n-1}$. Consequently as above the formula $\psi$ is infrafiltered. By Proposition 5 the formula $\varphi$ is infrafiltered too. So the assertion $A(n)$ holds.

By the complete induction principle the assertion $A(n)$ holds for every $n \in \omega_{0}$. This means that any formula $\varphi \in \Psi$ is infrafiltered.

Let $\varphi$ be an arbitrary formula of the language $L\left(\Sigma_{2}^{g}\right)$. Consider for $\varphi$ the accompanying formula $\varphi^{*}$ defined in section 4. By the definition of the operation $\varphi \mapsto \varphi^{*}$, we have $\varphi^{*} \in \Psi$. As proved above the formula $\varphi^{*}$ is infrafiltered, i.e., $\left\{g \in F \mid U_{g} \vDash \varphi^{*}\left[\gamma_{g}\right]\right\} \in \mathcal{D} \leftrightarrow U \vDash \varphi^{*}[\gamma]$. Proposition 1 implies the equivalences $U \vDash \varphi^{*}[\gamma] \leftrightarrow U \vDash \varphi[\gamma]$ and 
$U_{g} \vDash \varphi^{*}\left[\gamma_{g}\right] \leftrightarrow U_{g} \vDash \varphi\left[\gamma_{g}\right]$. In result we get the following chain of equivalences: $\left\{g \in F \mid U_{g} \vDash \varphi\left[\gamma_{g}\right]\right\} \in \mathcal{D} \leftrightarrow$ $\left\{g \in F \mid U_{g} \vDash \varphi^{*}\left[\gamma_{g}\right]\right\} \in \mathcal{D} \leftrightarrow U \vDash \varphi^{*}[\gamma] \leftrightarrow U \vDash \varphi[\gamma]$. It means that the formula $\varphi$ is infrafiltered.

This theorem has one important corollary. Let $\Phi$ be some set of formulas of the language $L\left(\Sigma_{2}^{g}\right)$ of the generalized second-order signature $\Sigma_{2}^{g}$. Let the set $\Phi$ has a model $\left(U_{0}, \gamma_{0}\right)$ of the signature $\Sigma_{2}^{g}$ with true generalized equalities and belongings. Take an arbitrary set $F$ and an arbitrary ultrafilter $\mathcal{D}$ on $F$. Consider the collection of the models $\left(\left(U_{f}, \gamma_{f}\right) \mid f \in F\right)$ such that $\left(U_{f}, \gamma_{f}\right) \equiv\left(U_{0}, \gamma_{0}\right)$. The infra-D -product infra-D $\mathcal{D}$-prod $\left(U_{f} \mid f \in F\right)$ of the collection $\left(U_{f} \mid f \in F\right)$ will be called the infra-D-power of the system $U_{0}$ with the exponent $F$ and will be denoted by infra-D-power $\left(U_{0}, F\right)$. The crossing $\bowtie\left(\gamma_{f} \mid f \in F\right)$ of the collection $\left(\gamma_{f} \mid f \in F\right)$ will be called the crossing of the evaluation $\gamma_{0}$ in the quantity $F$ and will be denoted by $\bowtie\left(\gamma_{0}, F\right)$.

Corollary Let $\Phi$ be some set of formulas of the language $L\left(\Sigma_{2}^{g}\right)$. If the set $\Phi$ has a model $\left(U_{0}, \gamma_{0}\right)$ of the signature $\Sigma_{2}^{g}$ with true generalized equalities and belongings, then for every set $F$ and every ultrafilter $\mathcal{D}$ on $F$ the set $\Phi$ has also the model (infra-D-Dower $\left.\left(U_{0}, F\right), \bowtie\left(\gamma_{0}, F\right)\right)$ of the signature $\Sigma_{2}^{g}$ with true generalized equalities and belongings.

It implies that if a set $\Phi$ of formulas of the language $L\left(\Sigma_{2}^{g}\right)$ has a model with true generalized equalities and belongings, then it has the same model of an arbitrary large power. Therefore the generalized second-order logic has the upper Lövengame-Skolem property, in contrast with the standard second-order logic, which does not have this property (see Mendelson, 1997, Appendix, (III)).

\section{Compactness Theorem for Formulas of the Language $\mathrm{L}\left(\Sigma_{2}^{g}\right)$ of the Generalized Second-Order Signature}

Theorem 2 Let $\Phi$ and $\Psi$ be some sets of formulas of the language $L\left(\Sigma_{2}^{g}\right)$ of the generalized second-order signature $\Sigma_{2}^{g}$. Let for every finite subset $f$ of the set $\Phi$ the set of formulas $f+(E 1-E 4)+\Psi$ has a model $\left(U_{f}, \gamma_{f}\right)$ of the signature $\Sigma_{2}^{\underline{g}}$ such that collection $\left(\left(U_{f}, \gamma_{f}\right) \mid f \in F\right)$ is pairwise $H$-concordant. Then the set of formulas $\Phi+(E 1-E 4)+\Psi$ has a model $(U, \gamma)$ of the signature $\Sigma_{2}^{g}$.

Proof. Consider the set $F \equiv\left\{f \subset \Phi|0<| f \mid<\omega_{0}\right\}$ of all finite non-empty subsets from $\Phi$.

For an element $f \in F$ consider the set $F_{f} \equiv\{g \in F \mid f \subset g\}$. Since $f \in F_{f}$, we have $F_{f} \neq \varnothing$. The set $\mathfrak{C} \equiv\left\{F_{f} \mid f \in F\right\}$ has the finite intersection property. So there is some ultrafilter $\mathcal{D}$ on the set $F$ including the set $\mathfrak{C}$.

Consider the system $U \equiv$ infra- $\mathcal{D}-\operatorname{prod}\left(U_{f} \mid f \in F\right)$, constructed in Section 6. Consider the evaluation $\gamma \equiv \bowtie$ $\left(\gamma_{f} \mid f \in F\right)$ on the system $U$, constructed in Section 6. By Lemma 2 from Section $6 U$ is a system with the true generalized equalities and belongings.

Prove that the evaluated system $(U, \gamma)$ is a model for the set $\Phi$.

Suppose $\varphi \in \Phi$. Consider the set $F_{\{\varphi\}}$. By condition, $U_{\{\varphi\}} \vDash \varphi\left[\gamma_{\{\varphi\}}\right]$. Consider the set $G_{\varphi} \equiv\left\{g \in F \mid U_{g} \vDash \varphi\left[\gamma_{g}\right]\right\}$. If $g \in F_{\{\varphi\}}$, then $\{\varphi\} \subset g$ implies $\varphi \in g$. Therefore $U_{g} \vDash \varphi\left[\gamma_{g}\right]$. Consequently, $F_{\{\varphi\}} \subset G_{\varphi}$. Since $F_{\{\varphi\}} \in \mathcal{D}$, we have $G_{\varphi} \in \mathcal{D}$.

By Theorem 1 from Section 7 we infer the property $U \vDash \varphi[\gamma]$. So $(U, \gamma)$ is a model for the set $\Phi$. The fact that $(U, \gamma)$ is a model for the set $\Psi$ follows immediately from Theorem 1.

\section{Uncountable Models of the Second-Order Generalized Peano-Landau Arithmetic}

At first we describe the Peano-Landau arithmetic in the generalized second-order language of the onegrade signature of the rank $1 \mid 0$.

Put $m=0$ and $n=0$, i.e., we shall consider the single basic first-order type of the form $\pi \equiv\langle 0,1\rangle$ without auxiliary first-order types. Consider the second-order types $\varkappa \equiv[\pi]$ and $\rho \equiv[\pi, \pi]$ and the type domain $\Theta \equiv \Theta_{A r 2}^{2} \equiv\{\pi, \varkappa, \rho\}$ of the rank $1 \mid 0$ with the belonging type subdomain $\Theta_{b} \equiv\{\varkappa, \rho\}$.

Put $\Omega_{\pi} \equiv 1, \Omega_{\varkappa} \equiv \varnothing, \Omega_{\rho} \equiv 1$, and consider collections $\Sigma_{c}^{\pi} \equiv\left(\sigma_{\omega}^{\pi} \mid \omega \in \Omega_{\pi}\right)=\sigma_{0}^{\pi}, \Sigma_{c}^{\varkappa} \equiv\left(\sigma_{\omega}^{\varkappa} \mid \omega \in \Omega_{\varkappa}\right)=\varnothing$, and $\Sigma_{c}^{\rho} \equiv\left(\sigma_{\omega}^{\rho} \mid \omega \in \Omega_{\rho}\right)=\sigma_{0}^{\rho}$. They compose the signature of constants of the type domain $\Theta$ of the form $\Sigma_{c} \equiv$ $\left(\Sigma_{c}^{\tau} \mid \tau \in \Theta\right)=\left(\sigma_{0}^{\pi}, \varnothing, \sigma_{0}^{\rho}\right)$ containing the constant $\sigma_{0}^{\pi}$, which is an objective first-order constant for denoting the natural number 0 , and the constant $\sigma_{0}^{\rho}$, which is a predicate second-order constant for expressing of the succession relation of Peano between a natural number $a$ and it's successor $a+1$.

Further along with $\sigma_{0}^{\pi}$ and $\sigma_{0}^{\rho}$ we shall simply write 0 and $\sigma$, respectively.

Take the signature of the generalized equalities of the type domain $\Theta$ of the form $\Sigma_{e} \equiv\left(\delta_{\tau} \mid \tau \in \Theta\right)=\left(\delta_{\pi}, \delta_{\varkappa}, \delta_{\rho}\right)$ containing the first-order equality $\delta_{\pi}$ and the second-order equalities $\delta_{[\pi]}$ and $\left.\delta_{[} \pi, \pi\right]$.

Take the signature of the generalized belongings of the type domain $\Theta$ of the form $\Sigma_{b} \equiv\left(\varepsilon_{\tau} \mid \tau \in \Theta_{b}\right)=\left(\varepsilon_{\varkappa}, \varepsilon_{\rho}\right)$. 
Finally, take a denumerable set $\Sigma_{v}^{\pi}$ of objective variables $x^{\pi}, y^{\pi}, \ldots$ of the first-order type $\pi$ and denumerable sets $\Sigma_{v}^{\varkappa}$ and $\Sigma_{v}^{\rho}$ of predicate variables $u^{\varkappa}, v^{\varkappa}, \ldots$ and $u^{\rho}, v^{\rho}, \ldots$ of the second-order types $\varkappa$ and $\rho$, respectively.

They form the signature $\Sigma_{v} \equiv\left(\Sigma_{v}^{\tau} \mid \tau \in \Theta\right)=\left(\Sigma_{v}^{\pi}, \Sigma_{v}^{\varkappa}, \Sigma_{v}^{\rho}\right)$ of variables of the type domain $\Theta$.

Consider the one grade generalized signature $\Sigma_{A r 2}^{g} \equiv \Sigma_{c}\left|\Sigma_{e}\right| \Sigma_{b} \mid \Sigma_{v}$ of the rank $1 \mid 0$ and its language $L\left(\Sigma_{A r 2}^{g}\right)$. Terms $p, q, r, \ldots$ of this language are constants and variables only, the atomic equality formulas have the forms $q^{\pi} \delta_{\pi} r^{\pi}$, $q^{\varkappa} \delta_{\varkappa} r^{\varkappa}$, and $q^{\rho} \delta_{\rho} r^{\rho}$. Respectively, the atomic belonging formulas have the forms $q^{\pi} \varepsilon_{\varkappa} r^{\varkappa}$ and $\left(p^{\pi}, q^{\pi}\right) \varepsilon_{\rho} r^{\rho}$.

Further along with $x^{\pi}, y^{\pi}, \ldots$ and $\delta_{\pi}$ we shall simply write $x, y, \ldots$ and $\delta$.

The axioms of the second-order generalized Peano-Landau arithmetic are the following ones:

A1) $\forall x_{1}, x_{2}, y\left(\left(x_{1}, y\right) \varepsilon_{\rho} \sigma \wedge\left(x_{2}, y\right) \varepsilon_{\rho} \sigma \Rightarrow x_{1} \delta x_{2}\right)$;

A2) $\forall x, y_{1}, y_{2}\left(\left(x, y_{1}\right) \varepsilon_{\rho} \sigma \wedge\left(x, y_{2}\right) \varepsilon_{\rho} \sigma \Rightarrow y_{1} \delta y_{2}\right)$;

A3) $\forall x, y\left((x, y) \varepsilon_{\rho} \sigma \Rightarrow \neg(y \delta 0)\right)$;

A4) $\forall u^{\varkappa}\left(0 \varepsilon_{\varkappa} u^{\varkappa} \wedge \forall x, y\left(x \varepsilon_{\varkappa} u^{\varkappa} \wedge(x, y) \varepsilon_{\rho} \sigma \Rightarrow y \varepsilon_{\varkappa} u^{\varkappa}\right) \Rightarrow \forall z\left(z \varepsilon_{\varkappa} u^{\varkappa}\right)\right)$.

Consider the following generalized extensionality properties:

$P E$ 1) $\forall u^{\varkappa}, v^{\varkappa}\left(u^{\varkappa} \delta_{\varkappa} v^{\varkappa} \Leftrightarrow \forall x\left(x \varepsilon_{\varkappa} u^{\varkappa} \Leftrightarrow x \varepsilon_{\varkappa} v^{\varkappa}\right)\right)$;

$P E$ 2) $\forall u^{\rho}, v^{\rho}\left(u^{\rho} \delta_{\rho} v^{\rho} \Leftrightarrow \forall x, y\left((x, y) \varepsilon_{\rho} u^{\rho} \Leftrightarrow(x, y) \varepsilon_{\rho} v^{\rho}\right)\right)$.

Consider the set $\mathbb{N}_{0} \equiv \omega_{0}$ of all natural numbers, which was constructed in the theory NBG of sets and classes.

For the formation $\mathbb{N}_{0}$ of the rank $1 \mid 0$ and of the signature $\Sigma_{A r 2}^{g}$ consider the following collections $S_{c}^{\pi} \equiv\left(s_{\omega}^{\pi} \mid \omega \in\right.$ $\left.\Omega_{\pi}\right)=s_{0}^{\pi}, S_{c}^{\varkappa} \equiv\left(s_{\omega}^{\varkappa} \mid \omega \in \Omega_{\varkappa}\right)=\varnothing$, and $S_{c}^{\rho} \equiv\left(s_{\omega}^{\rho} \mid \omega \in \Omega_{\rho}\right)=s_{0}^{\rho}$. They compose the collection of constant structures $S_{c} \equiv\left(S_{c}^{\tau} \mid \tau \in \Theta\right)=\left(s_{0}^{\pi}, \varnothing, s_{0}^{\rho}\right)$, containing the constant structure $s_{0}^{\pi} \in \pi\left(\mathbb{N}_{0}\right)=\mathbb{N}_{0}$, which is the initial natural number, and the constant structure $s_{0}^{\rho} \in \rho\left(\mathbb{N}_{0}\right)=\mathcal{P}\left(\mathbb{N}_{0} \times \mathbb{N}_{0}\right)$, which is the set of all pairs of natural numbers of the form $\langle a, a+1\rangle$.

Further along with $s_{0}^{\pi}$ and $s_{0}^{\rho}$ we shall write simply 0 and $s$, respectively.

Consider the collection of the equality relations of the form $S_{e} \equiv\left(\approx_{\tau} \mid \tau \in \Theta\right)=\left(\approx_{\pi}, \approx_{\varkappa}, \approx_{\rho}\right) \equiv\left(=\left|\mathbb{N}_{0}^{2},=\right| \mathcal{P}\left(\mathbb{N}_{0}\right)^{2},=\right.$ $\left.\mathcal{P}\left(\mathbb{N}_{0} \times \mathbb{N}_{0}\right)^{2}\right)$, containing in the capacity of the first-order equality relation $\approx_{\pi}$ and of the second-order equality relations $\approx_{\varkappa}$ and $\approx_{\rho}$ the restrictions on the indicated sets one and the same set-theoretical equality $=$ in the theory NBG.

Consider the collection of the belonging correspondences of the form $S_{b} \equiv\left(\leftarrow_{\tau} \mid \tau \in \Theta\right)=\left(\leftarrow_{\varkappa}, \leftarrow_{\rho}\right) \equiv(\epsilon$ $\left.\left|\mathbb{N}_{0} \times \mathcal{P}\left(\mathbb{N}_{0}\right), \in\right|\left(\mathbb{N}_{0} \times \mathbb{N}_{0}\right) \times \mathcal{P}\left(\mathbb{N}_{0} \times \mathbb{N}_{0}\right)\right)$, containing in the capacity of the belonging correspondences $<_{\varkappa}$ and $<_{\rho}$ the restrictions on the indicated sets one and the same set-theoretical belonging correspondence $\in$ in the theory NBG.

Finally, take the collection of the terminals over the formation $\mathbb{N}_{0}$ of the form $S_{v} \equiv\left(\tau\left(\mathbb{N}_{0}\right) \mid \tau \in \Theta\right)=\left(\pi\left(\mathbb{N}_{0}\right), \varkappa\left(\mathbb{N}_{0}\right)\right.$, $\left.\rho\left(\mathbb{N}_{0}\right)\right)=\left(\mathbb{N}_{0}, \mathcal{P}\left(\mathbb{N}_{0}\right), \mathcal{P}\left(\mathbb{N}_{0} \times \mathbb{N}_{0}\right)\right)$.

These collections compose the one grade superstructure $S_{A r 2} \equiv\left(S_{c}, S_{e}, S_{b}, S_{v}\right)$ of the signature $\Sigma_{A r 2}^{g}$ of the rank $1 \mid 0$ over the formation $\mathbb{N}_{0}$.

The system $A r 2 \equiv\left(\mathbb{N}_{0}, S_{A r 2}\right)$ of the signature $\Sigma_{A r 2}^{g}$ can be called the natural series of Peano-Landau of the secondorder in the set theory $N B G$, because it models the following Peano-Landau postulates:

P1) $\forall a_{1}, a_{2}, b\left(\left\langle a_{1}, b\right\rangle \in s \wedge\left\langle a_{2}, b\right\rangle \in s \Rightarrow a_{1}=a_{2}\right)$;

P2) $\forall a, b_{1}, b_{2}\left(\left\langle a, b_{1}\right\rangle \in s \wedge\left\langle a, b_{2}\right\rangle \in s \Rightarrow b_{1}=b_{2}\right)$;

P3) $\forall a, b(\langle a, b\rangle \in s \Rightarrow b \neq 0)$;

P4) $\forall P(0 \in P \wedge \forall a, b(a \in P \wedge\langle a, b\rangle \in s \Rightarrow b \in P) \Rightarrow \forall c(c \in P))$.

Consider an evaluation $\gamma$ on the system $A r 2$ such that $\gamma(x) \in \pi\left(\mathbb{N}_{0}\right)=\mathbb{N}_{0}, \gamma\left(u^{\varkappa}\right) \in \varkappa\left(\mathbb{N}_{0}\right)=\mathcal{P}\left(\mathbb{N}_{0}\right)$, and $\gamma\left(u^{\rho}\right) \in$ $\rho\left(\mathbb{N}_{0}\right)=\mathcal{P}\left(\mathbb{N}_{0} \times \mathbb{N}_{0}\right)$.

For the evaluated system $(A r 2, \gamma)$ the following assertion holds.

Lemma 8 The evaluated system (Ar2, $\gamma$ ) is the standard model for the set of formulas E1, E2, E3, E4, A1, A2, A3, A4, PE1, and PE2 of the language $L\left(\Sigma_{A r 2}^{g}\right)$. 
Proof. The satisfactions $A r 2 \vDash A 1[\gamma], A r 2 \vDash A 2[\gamma], A r 2 \vDash A 3[\gamma]$, and $A r 2 \vDash A 4[\gamma]$ follow from the correctness for the system $A r 2$ of Peano-Landau postulates $P 1, P 2, P 3$, and $P 4$, respectively. The other satisfactions are checked immediately.

Therefore the evaluated system $(A r 2, \gamma)$ can be called the generalized natural series of Peano-Landau of the second-order in the set theory $N B G$.

Now construct an uncountable model. Take an arbitrary set $F$ and an arbitrary ultrafilter $\mathcal{D}$ on $F$. Consider the system infra-D-power $(A r 2, F)$ and the evaluation $\bowtie(\gamma, F)$, defined in section 7 .

Theorem 3 The evaluated system (infra-D-power $(A r 2, F), \bowtie(\gamma, F)$ ) is the generalized model for the set of formulas E1, E2, E3, E4, A1, A2, A3, A4, PE1, and PE2 of the language $L\left(\Sigma_{A r 2}^{g}\right)$. The support of the model is the Baire set $\mathbb{N}_{0}^{F}$. If $|F| \geq \omega_{0}$, then the support is uncountable.

Proof. The assertion follows from the preceding lemma and Theorem 1.

\section{Conclusion}

The compactness theorem for the generalized second-order logic allows us to build unusual, but interesting models of such classical theories as the arithmetic, the theory of real numbers, and so on.

For example, if we shall add to the axioms of Section 9 the countable set of axioms $\exists x^{\tau} \neg(x \delta \bar{n})$, where $\bar{n}$ denotes the $n$-th numeral (see Mendelson, 1997, p. 160), then using the compactness theorem from Section 8 we can construct a generalized model of the second-order generalized Peano-Landau arithmetic with infinite elements.

For the similar set of axioms there exists a generalized model of the generalized second-order theory of real numbers with infinitely large and infinitely small elements.

The limited frames of the article did not allow the authors to present these ideas in more details. However they illustrate that the material, presented in the given paper, shows a new interesting direction in constructing some generalized models of classical second-order theories.

\section{References}

Boolos, G., Burgess, J. P., \& Jeffrey, R. (2007). Computability and logic (3rd ed.). Cambridge, UK: University Press. http://dx.doi.org/10.1017/CBO9780511804076

Ershov, Yu. L., \& Palyutin, E. A. (1984). Mathematical logic. Translated from the Russian by V. Shokyrov. Moscow: Mir publishers.

Loś, J. (1955). Quelques remarques, théorems et problèms sur les classes définisables d'algèbres. In Mathematical Interpretations of Formal Systems (pp. 98-113). Amsterdam: North-Holland Publishing Company.

Maltsev, A. I. (1973). Algebraic systems. Berlin: Springer.

Mendelson, E. (1997). Introduction to Mathematical Logic. Chapman \& Hall/CRC.

Rossberg, M. (2004). First-Order and Second-Order logic and Completeness. In Hendricks et al. (Eds.), FirstOrder Logic Revisited. Berlin: Logos Verlag.

Shapiro, S. (1991). Foundations without Foundationalism: A Case for Second-Order Logic. Oxford: Oxford University Press.

Shoenfield, J. R. (2001). Mathematical logic. A K Peters.

Takeuti, G. (2013). Proof theory. Mineola, NY: Dover Publications.

Tourlakis, G. (2003). Lectures in logic and set theory. Cambridge, UK: Cambridge University Press.

Van Dalen, D. (1997). Logic and Structure. Berlin: Springer-Verlag.

Väänänen, J. (2001). Bulletin of Symbolic Logic, 7(4), 504-520. http://dx.doi.org/10.2307/2687796

Zakharov, V. K. (2008). Compactness theorem for some generalized second-order language. In E. S. Polovinkin et al. (Eds.), Modern problems of fundamental and applied mathematics (In Russian, pp. 11-31). Transactions. M.: Moscow Institute of Physics and Technology (State University). 


\section{Notes}

Note 1. Note that use a generalized belonging was explored in the forcing method in the form $x \in \in_{p} y$ (see e.g., Shoenfield, 1967, 9.8).

\section{Copyrights}

Copyright for this article is retained by the author(s), with first publication rights granted to the journal.

This is an open-access article distributed under the terms and conditions of the Creative Commons Attribution license (http://creativecommons.org/licenses/by/3.0/). 